\title{
Synthesis of Novel D-Glucose-derived Benzyl and Alkyl 1,2,3-Triazoles as Potential Antifungal and Antibacterial Agents
}

\author{
Jin-Jian Wei, Lei Jin, ${ }^{\dagger}$ Kun Wan, and Cheng-He Zhou* \\ Laboratory of Bioorganic \& Medicinal Chemistry, School of Chemistry and Chemical Engineering, Southwest University, \\ Chongqing 400715, P. R. China.*E-mail: zhouch@swu.edu.cn \\ ${ }^{\dagger}$ School of Pharmaceutical Sciences, Southwest University, Chongqing 400715, P. R. China \\ Received May 17, 2010, Accepted November 15, 2010
}

\begin{abstract}
A series of novel glucose derived benzyl and alkyl 1,2,3-triazoles and their hydrochlorides have been synthesized via $\mathrm{Cu}(\mathrm{I})$-catalyzed 1,3-dipolar cycloaddition. All the new compounds were characterized by MS, IR and NMR spectra. The DEPT, APT, ${ }^{1} \mathrm{H}-{ }^{1} \mathrm{H}$ and ${ }^{1} \mathrm{H}-{ }^{13} \mathrm{C}$ 2D NMR spectra for some compounds were also recorded. These compounds were evaluated for their in vitro antibacterial activities against Staphylococcus aureus ATCC 29213, Bacillus subtilis, Bacillus proteus, Pseudomonas aeruginosa, Escherichia coli ATCC 25922, and antifungal activities against Candida albicans and Aspergillus fumigatus. The bioactive data revealed that (3R,4S,5S,6S)-2-(hydroxymethyl)-6-methoxy4,5-bis((1-octyl-1H-1,2,3-triazol-4-yl)methoxy)-tetrahydro-2H-pyran-3-ol 8a exhibited excellent antifungal activity against $A$. fumigatus with an MIC value of $0.055 \mathrm{mM}$ compared to Fluconazole. It also showed broad inhibitory efficacy against tested bacterial strains with MIC values ranging from $0.049 \mathrm{mM}$ to $0.39 \mathrm{mM}$.
\end{abstract}

Key Words: 1,2,3-Triazole, Glucose, Antibacterial, Antifungal

\section{Introduction}

The alarming rates of emerging and reemerging microbial threats coupled with the growing emergence of antimicrobial resistance in hospitals are major concerns to the public health and scientific communities worldwide, especially in regard to multi-drug-resistant Gram-positive bacteria such as Methicillinresistant Staphylococcus aureus (MRSA). ${ }^{1}$ Thus, these trends have emphasized the urgent need for designing and developing new classes of antimicrobial agents with different chemical structures and mechanism of action compared with traditional drugs to improve their activities while retaining good bioavailability and safety profiles.

1,2,3-Triazole derivatives as an important class of heterocyclic compounds possessing myriad potential applications in chemical synthesis, material science and biology, have aroused progressive attention in recent years with the introduction of 'click chemistry' for their easy and efficient synthesis. ${ }^{2-5}$ In particular, 1,2,3-triazole serving as a potential pharmacophore, which was found to be potent antineoplasic, ${ }^{6}$ insecticidal, ${ }^{7}$ antibacterial, ${ }^{8}$ antifungal, ${ }^{9}$ antitubercular ${ }^{10}$ and anti-HIV agents, ${ }^{11,12}$ has occupied an important position in medicinal chemistry owing to its chemotherapeutic value. ${ }^{13,14}$ Some 1,2,3-triazole derivatives were used as DNA cleaving agents, ${ }^{15}$ potassium channel activators ${ }^{16}$ and cannabinoid CB1 receptors ${ }^{17}$ and so on. It is especially noteworthy that 1,2,3-triazole derivatives may be considered as new entry to azole antifungal agents. ${ }^{9}$

Although 1,2,3-triazole was not present in nature products, it could not be cleaved hydrolytically and was stable to metabolic degradation. Literature revealed that 1,4-substituted 1,2,3triazole had large dipole moment, which made it functionalized as a weak hydrogen bond donor and N-2, N-3 acting as good H-bond acceptors. ${ }^{18}$ Recent studies in 1,2,3-triazoles demonstrated that the hydrogen bonding and dipole interactions of the triazole core could favor in binding of biomolecular targets and improving solubility. ${ }^{19}$ Moreover, 1,2,3-triazole as an attractive bridge group, which could connect with two pharmacophores to give an innovative bifunctional active molecule, has become increasingly useful and important in constructing bioactive molecules and functional molecules. ${ }^{13}$ Noticeably, the bioisosteric replacement between 1,2,3-triazole and its bioisoster 1,2,4-triazole, amide, or even other nitrogen-containing aromatic heterocycles is of special interest in medicinal chemistry, which represents an efficient concept for the discovery and development of novel triazole drugs. ${ }^{20,21}$ In addition, many investigations demonstrated that biological activity of azole derivatives was significantly influenced by the introduction of variable aromatic substituents. Especially some halogen-substituted or strong electron-withdrawing substituted aromatic compounds which were linked to azoles, were found to increase lipid solubility to a certain extent and could efficiently enhance the antimicrobial activity. ${ }^{22,23}$ The fluoro or chloro substituted aromatic rings represented a valuable strategy and were extensively employed in drug discovery. Meanwhile, the introduction of alkyl moiety into various heterocyclic systems as the large non-polar portion could play an important role in modulating the physicochemical properties of the whole molecule that possibly avoid some side effects and improve their pharmacokinetic and pharmacodynamic behaviors. This was also helpful to increase their biological activities. ${ }^{24-26}$

On the basis of all above observations and considering that sugar moiety with polyhydroxyl groups has been extensively employed in drug design, frequently used to improve water solubility and usually introduced into artificial receptors to increase the interaction between receptors and guests for molecular recognition, ${ }^{27-29}$ a series of novel D-glucose-derived 1,2,3triazoles bearing alkyl chain with different length and aralkyl group with various substituted phenyl rings were designed and 

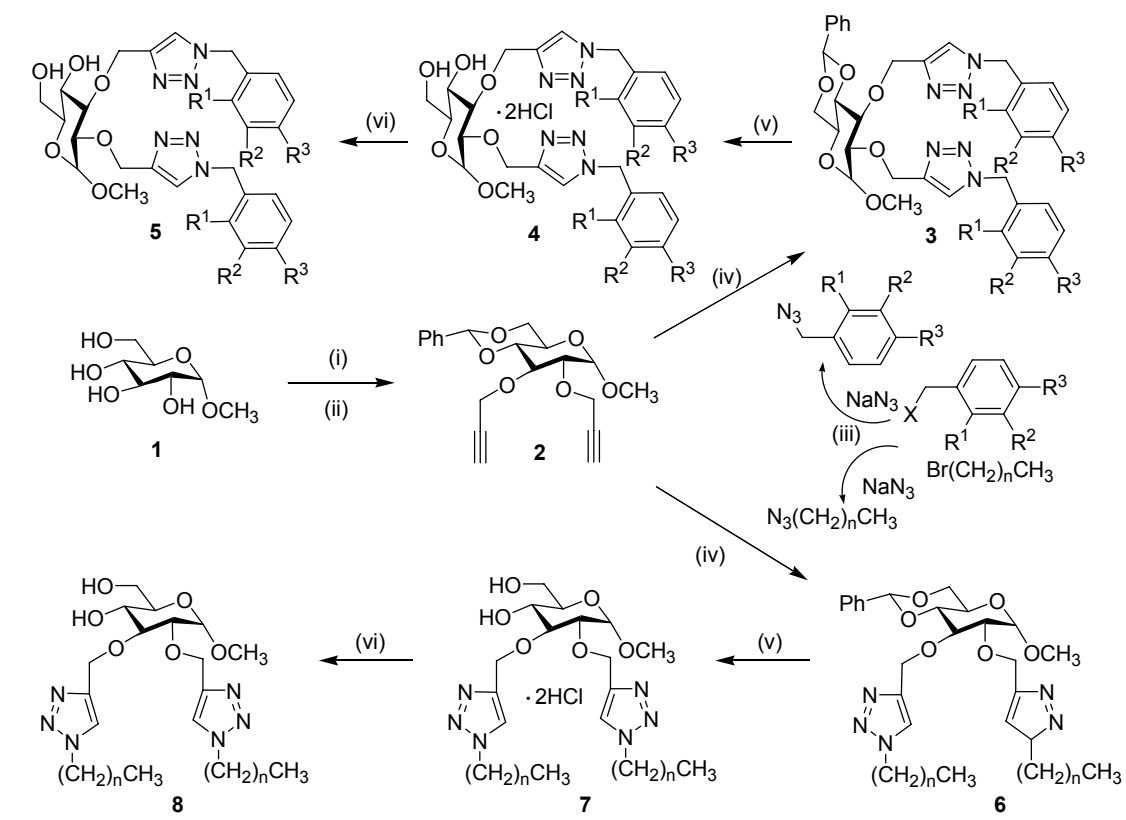

3-5: $\quad$ a $\mathrm{R}^{1}=\mathrm{Cl}, \mathrm{R}^{2}=\mathrm{H}, \mathrm{R}^{3}=\mathrm{H} ; \quad$ b $\mathrm{R}^{1}=\mathrm{Cl}, \mathrm{R}^{2}=\mathrm{H}, \mathrm{R}^{3}=\mathrm{Cl}$; $\mathrm{R}^{1}=\mathrm{H}, \mathrm{R}^{2}=\mathrm{Cl}, \mathrm{R}^{3}=\mathrm{Cl}$; d $R^{1}=H, R^{2}=C l, R^{3}=H ; \quad$ e $R^{1}=H, R^{2}=H, R^{3}=C l ; f R^{1}=F, R^{2}=H, R^{3}=F$; g $R^{1}=H, R^{2}=H, R^{3}=N_{2} ; \quad h R^{1}=H, R^{2}=H, R^{3}=F ; \quad i R^{1}=H, R^{2}=H, R^{3}=B r$

Reagents and conditions: (i) $\mathrm{PhCHO} / \mathrm{ZnCl}_{2}$; (ii) $\mathrm{NaH} /$ abs. THF, propargyl bromide; (iii) $\mathrm{CH}_{3} \mathrm{COCH}_{3} / \mathrm{H}_{2} \mathrm{O}=1 / 1$ (v/v) sodium azide (1.5 equiv), $60{ }^{\circ} \mathrm{C}$; (iv) benzyl or alkyl azides (2 equiv), $\mathrm{CuSO}_{4} \cdot 5 \mathrm{H}_{2} \mathrm{O}$ ( 0.1 equiv), sodium ascorbate $\left(0.2\right.$ equiv), $\mathrm{DMSO} / \mathrm{H}_{2} \mathrm{O}=4 / 1$ ( $\left.\mathrm{v} / \mathrm{v}\right)$; (v) $5 \%$ hydrochloric acid; (vi) $25 \% \mathrm{NH}_{3}$ aq.

Scheme 1. Synthetic route of novel glucose-derived 1,2,3-triazoles 5a-i and 8a-b

synthesized in this contribution. All these newly synthesized compounds were evaluated for their antibacterial and antifungal activities. Incorporation of glucose was helpful to improve the water solubility of target molecules. Various substituted benzyl and alkyl groups were introduced into the target compounds in order to investigate their structure-activity relationships.

\section{Results and Discussion}

Chemistry. Compounds 5a-i and 8a-b were synthesized in high yields ranging from $64 \%$ to $99 \%$ by the cycloaddition of alkyne compound $\mathbf{2}$ with benzyl or alkyl azides using catalytic amount of copper sulfate and sodium ascorbate in $t-\mathrm{BuOH} /$ $\mathrm{H}_{2} \mathrm{O}$ at room temperature. Accordingly, the intermediate alkyl azides were prepared from commercially available benzyl or alkyl bromides. Alkyl bromides treated with excess $\mathrm{NaN}_{3}(1.5$ equiv) in acetone and water at $60{ }^{\circ} \mathrm{C}$ could give alkyl azides almost quantitatively. Compound 2 was synthesized in $81 \%$ yield by the reaction of methyl 4,6-O-benzylidene- $\alpha$-D-glucopyranoside with propargyl bromide in the presence of $\mathrm{NaH}$ using anhydrous THF as solvent. In the propargylation of methyl 4,6-O-benzylidene $\alpha$-D-glucopyranoside, the reaction temperature, dropwise rate and dosage of propargyl bromide as well as the nature of solvent exerted great influence on the reaction. Generally, slow addition of excess propargyl bromide (six equivalent of protected $\alpha$-D-glucopyranoside) at low temperature such as ice-salt bath together with assurance of an anhydrous and anaerobic system facilitated the reaction and raised the yields apparently. No mono-propargyl- $\alpha$-D-glucoside was obtained. Either the temperature higher than $50{ }^{\circ} \mathrm{C}$ or addition of all propargyl bromide at once resulted in a very complex system, which was difficult to separate. On the other hand, the effect of solvent was another quite important factor in the propargylation of glucoside. Our observation indicated that anhydrous aprotic solvent THF could give good reactivity and excellent yields.

Analyses of spectra. The structures of all the synthesized compounds 5a-i and 8a-b were in accordance with their spectral analyses. MS of compound $\mathbf{3 f}$ displayed $[\mathrm{M}+\mathrm{H}]^{+}$peak corresponding to its molecular weight. Typical fragment ions such as $\left[\mathrm{M}-\mathrm{CH}_{3} \mathrm{O}\right]^{+}$and $\left[\mathrm{M}-\mathrm{PhCHO}-\mathrm{CH}_{3} \mathrm{O}\right]^{+}$were also observed. Besides, triazole hydrochlorides $\mathbf{4 a - i}$ as well as $\mathbf{7 a}$ and $\mathbf{7 b}$ gave $\mathrm{a}[\mathrm{M}+\mathrm{Na}-2 \mathrm{HCl}]^{+}$fragment ion peak in the mass spectra. The IR spectra of compound $\mathbf{3 f}$ exhibited two characteristic strong absorption bands at 3122 and $3079 \mathrm{~cm}^{-1}$ which were respectively attributed to the stretching vibration of triazole- $\mathrm{H}$ and Ar-H.

In the ${ }^{1} \mathrm{H}$ NMR spectrum of compound $\mathbf{3 f}$, triazole protons appeared as two distinct singlets at $\delta 7.78$ and $\delta 7.62 \mathrm{ppm}$, which indicated the different chemical environment of two triazole rings resulting from the chiral glucose. Electronic effects resulted in the large downfield of triazole 5-H linked glucoside $\mathrm{C}-3$ chain. From the ${ }^{13} \mathrm{C}$ NMR spectrum of compound $\mathbf{3 f}$, two sets of signals of triazole C-4 at $\delta 146.02$ and 144.35 ppm or triazole C-5 at $\delta 123.31$ and 122.90 ppm accounted the above spatial influence of two triazole rings. Also, the ${ }^{1} \mathrm{H}$ NMR spectrum of compound 3f showed a singlet (integration: three hydrogens) at $\delta 5.57 \mathrm{ppm}$ due to the presence of benzylidene group 
Table 1. The $\delta$ value of ${ }^{1} \mathrm{H}$ NMR and ${ }^{13} \mathrm{C}$ NMR in the glucoside of $\mathbf{3 f}$

\begin{tabular}{cccccccc}
\hline No & 1 & 2 & 3 & 4 & 5 & 6 & $\mathrm{OCH}_{3}$ \\
\hline$\delta_{\mathrm{H}}(\mathrm{ppm})$ & 4.80 & 3.60 & 3.93 & 3.56 & 3.80 & $4.27\left(\mathrm{H}_{\mathrm{eq}}\right), 3.72\left(\mathrm{H}_{\mathrm{ax}}\right)$ & 3.37 \\
Peak & $\mathrm{d}$ & $\mathrm{dd}$ & $\mathrm{t}$ & $\mathrm{t}$ & $\mathrm{ddd}$ & $\mathrm{dd}\left(\mathrm{H}_{\mathrm{eq}}\right), \mathrm{t}\left(\mathrm{H}_{\mathrm{ax}}\right)$ & $\mathrm{s}$ \\
$\delta_{\mathrm{C}}(\mathrm{ppm})$ & 98.70 & 79.48 & 78.30 & 81.87 & 62.30 & 69.06 & 55.22 \\
\hline
\end{tabular}

Table 2. In vitro antimicrobial activity of compounds $\mathbf{3 - 8 ^ { a , b , c }}$

\begin{tabular}{|c|c|c|c|c|c|c|c|}
\hline \multirow[b]{2}{*}{ Compd } & \multicolumn{7}{|c|}{$\mathrm{MIC} \mathrm{mM}(\mu \mathrm{g} / \mathrm{mL})$} \\
\hline & $\begin{array}{c}\text { C. albicans } \\
\text { ATCC } 76615\end{array}$ & A. fumigatus & $\begin{array}{c}\text { S. aureus } \\
\text { ATCC } 29213\end{array}$ & P. aeruginosa & B. subtilis & B. proteus & $\begin{array}{c}\text { E. coli } \\
\text { ATCC } 25922\end{array}$ \\
\hline $3 a$ & $0.74(512)$ & $0.74(512)$ & $0.74(512)$ & $0.74(512)$ & $0.74(512)$ & $0.74(512)$ & $0.74(512)$ \\
\hline $\mathbf{3 b}$ & $0.67(512)$ & $0.67(512)$ & $0.67(512)$ & $0.67(512)$ & $0.67(512)$ & $0.67(512)$ & $0.67(512)$ \\
\hline $3 \mathrm{c}$ & $0.67(512)$ & $0.67(512)$ & $0.67(512)$ & $0.67(512)$ & $0.67(512)$ & 0.17 & $0.67(512)$ \\
\hline 3d & $0.74(512)$ & $0.74(512)$ & $0.74(512)$ & $0.74(512)$ & $0.74(512)$ & $0.74(512)$ & $0.74(512)$ \\
\hline $3 e$ & $0.74(512)$ & $0.74(512)$ & $0.37(256)$ & $0.74(512)$ & $0.74(512)$ & $0.74(512)$ & $0.74(512)$ \\
\hline $3 f$ & $0.74(512)$ & $0.74(512)$ & $0.74(512)$ & $0.74(512)$ & $0.74(512)$ & $0.74(512)$ & $0.74(512)$ \\
\hline $3 g$ & $0.72(512)$ & $0.72(512)$ & $0.72(512)$ & $0.72(512)$ & $0.72(512)$ & $0.72(512)$ & $0.72(512)$ \\
\hline $3 \mathbf{h}$ & $0.78(512)$ & $0.78(512)$ & $0.39(256)$ & $0.78(512)$ & $0.78(512)$ & $0.78(512)$ & $0.78(512)$ \\
\hline $3 \mathbf{i}$ & $0.65(512)$ & $0.65(512)$ & $0.65(512)$ & $0.65(512)$ & $0.65(512)$ & $0.65(512)$ & $0.65(512)$ \\
\hline $4 a$ & $0.76(512)$ & $0.76(512)$ & $0.76(512)$ & $0.76(512)$ & $0.76(512)$ & $0.76(512)$ & $0.76(512)$ \\
\hline $4 b$ & $0.69(512)$ & $0.69(512)$ & $0.69(512)$ & $0.69(512)$ & $0.34(256)$ & $0.17(128)$ & $0.69(512)$ \\
\hline $4 c$ & $0.086(64)$ & $0.69(512)$ & $0.69(512)$ & $0.69(512)$ & $0.34(256)$ & $0.34(256)$ & $0.043(32)$ \\
\hline $4 d$ & $0.047(32)$ & $0.76(512)$ & $0.76(512)$ & $0.76(512)$ & $0.76(512)$ & $0.76(512)$ & $0.094(64)$ \\
\hline $4 e$ & $0.38(256)$ & $0.38(256)$ & $0.76(512)$ & $0.76(512)$ & $0.38(256)$ & $0.38(256)$ & $0.38(256)$ \\
\hline $4 f$ & $0.75(512)$ & $0.75(512)$ & $0.75(512)$ & $0.75(512)$ & $0.75(512)$ & $0.75(512)$ & $0.75(512)$ \\
\hline $4 g$ & $0.73(512)$ & $0.73(512)$ & $0.73(512)$ & $0.73(512)$ & $0.73(512)$ & $0.73(512)$ & $0.73(512)$ \\
\hline $4 h$ & $0.79(512)$ & $0.79(512)$ & $0.79(512)$ & $0.79(512)$ & $0.79(512)$ & $0.79(512)$ & $0.79(512)$ \\
\hline $4 i$ & $0.67(512)$ & $0.67(512)$ & $0.67(512)$ & $0.67(512)$ & $0.67(512)$ & $0.67(512)$ & $0.67(512)$ \\
\hline $5 a$ & $0.85(512)$ & $0.85(512)$ & $0.85(512)$ & $0.85(512)$ & $0.85(512)$ & $0.85(512)$ & $0.85(512)$ \\
\hline $5 b$ & $0.095(64)$ & $0.76(512)$ & $0.76(512)$ & $0.38(256)$ & $0.38(256)$ & $0.76(512)$ & $0.38(256)$ \\
\hline $5 c$ & $0.76(512)$ & $0.76(512)$ & $0.76(512)$ & $0.76(512)$ & $0.38(256)$ & $0.76(512)$ & $0.76(512)$ \\
\hline $5 d$ & $0.85(512)$ & $0.85(512)$ & $0.85(512)$ & $0.85(512)$ & $0.85(512)$ & $0.85(512)$ & $0.85(512)$ \\
\hline $5 e$ & $0.85(512)$ & $0.85(512)$ & $0.85(512)$ & $0.85(512)$ & $0.85(512)$ & $0.85(512)$ & $0.85(512)$ \\
\hline $5 f$ & $0.84(512)$ & $0.84(512)$ & $0.84(512)$ & $0.84(512)$ & $0.84(512)$ & $0.84(512)$ & $0.84(512)$ \\
\hline $5 h$ & $0.89(512)$ & $0.89(512)$ & $0.89(512)$ & $0.89(512)$ & $0.89(512)$ & $0.89(512)$ & $0.89(512)$ \\
\hline $5 \mathbf{i}$ & $0.74(512)$ & $0.74(512)$ & $0.74(512)$ & $0.74(512)$ & $0.74(512)$ & $0.74(512)$ & $0.74(512)$ \\
\hline $6 a$ & $0.77(512)$ & $0.77(512)$ & $0.77(512)$ & $0.77(512)$ & $0.77(512)$ & $0.77(512)$ & $0.77(512)$ \\
\hline $6 b$ & $0.66(512)$ & $0.66(512)$ & $0.66(512)$ & $0.66(512)$ & $0.66(512)$ & $0.66(512)$ & $0.66(512)$ \\
\hline $7 \mathbf{a}$ & $0.39(256)$ & $0.20(128)$ & $0.39(256)$ & $0.049(32)$ & $0.39(256)$ & $0.20(128)$ & $0.098(64)$ \\
\hline $7 b$ & $0.70(512)$ & $0.70(512)$ & $0.70(512)$ & $0.70(512)$ & $0.70(512)$ & $0.70(512)$ & $0.70(512)$ \\
\hline $8 a$ & $0.22(128)$ & $0.055(32)$ & $0.055(32)$ & $0.055(32)$ & $0.44(256)$ & $0.055(32)$ & $0.11(64)$ \\
\hline $8 b$ & $0.74(512)$ & $0.74(512)$ & $0.74(512)$ & $0.74(512)$ & $0.74(512)$ & $0.74(512)$ & $0.74(512)$ \\
\hline $\mathbf{A}$ & $0.052(16)$ & $1.67(512)$ & - & - & - & - & - \\
\hline B & - & - & $1.59(512)$ & $1.59(512)$ & 1.59 (512) & $0.05(16)$ & $0.05(16)$ \\
\hline
\end{tabular}

${ }^{a}$ Minimum inhibitory concentrations were determined by micro broth dilution method for microdilution plates. ${ }^{b} \mathbf{A}=$ Fluconazole, $\mathbf{B}=$ Chloramphenicol. ${ }^{c}$ C. albicans ATCC76615, Candida albicans ATCC76615; A. fumigatus, Aspergillus fumigatus; S. aureus ATCC 29213, Staphylococcus aureus ATCC 29213; P. aeruginosa, Pseudomonas aeruginosa; B. proteus, Bacillus proteus; B. subtilis, Bacillus subtilis; E. coli ATCC 25922, Escherichia coli ATCC 25922.

and $\mathrm{CH}_{2}$ linked with 2-O moiety. It could be confirmed by the correlation of two carbon at $101.52(\mathrm{Ph} \mathrm{CH}) \mathrm{ppm}$ and 47.08 $\left(\mathrm{OCH}_{2}\right)$ ppm with the hydrogen in $5.52 \mathrm{ppm}$ from the gHSQC 2D NMR. Similar patterns were observed from ${ }^{1} \mathrm{H}$ NMR and ${ }^{13} \mathrm{C}$ NMR spectra for compounds 3a-i. The $\delta$ value of ${ }^{1} \mathrm{H}$ NMR and ${ }^{13} \mathrm{C}$ NMR in the glucoside can also be concluded from the gCOSY and gHSQC (see Table 1).

From the NMR spectrum of compound $\mathbf{5 f}$, the carbon and proton singlets of $\mathrm{Ph}-\mathrm{CH}$ disappeared, which appeared at $\delta$
101.52 and $5.57 \mathrm{ppm}$ in compound $\mathbf{3 f}$. This indicated the complete deprotection of benzaldehyde. Besides, a singlet in 5.10 ppm (integration: four hydrogens) showing no correlation in gCOSY and gHSQC can be ascribed to the presence of $\mathrm{OH}$, which was influenced by the association with water or the hydrogen bond with fluorine. The proton of triazole ring in hydrochlorides 4a-i showed large downfield shift of 0.3 - 0.9 ppm compared to the protected $\alpha$-D-glucopyranoside derived bis-triazoles 3a-i, ascribed to the presence of positive charges in the 
triazole system. All the anomeric 1-H signals in compounds 5a-i gave small coupling constants $J$ values $(2.9-3.6 \mathrm{~Hz})$. This demonstrated that these D-glucose derivatives possessed $\alpha$ configuration. Thus, substitutions have not resulted in a change of the $\alpha$-D-glucoside configuration.

Pharmacology. The prepared compounds (3-8) were evaluated for their antimicrobial activity in vitro against $S$. aureus, B. subtilis as Gram-positive, E. coli, P. aeruginosa, B. proteus as Gram-negative bacteria and $C$. albicans, $A$. fumigatus as fungi by broth microdilution assay method. ${ }^{30}$ The minimum inhibitory concentrations were determined and compared with Chloramphenicol and Fluconazole as standard drugs. The minimum inhibitory concentration (MIC) was given in Table 2 .

Antifungal activity: The investigation of antifungal screening data indicated that some of the newly synthesized compounds showed potent activity in vitro. As seen in Table 2, 2,4-dichloro substituted benzyl 1,2,3-triazole $\mathbf{5} \mathbf{b}$ as well as the hydrochlorides $\mathbf{4 c}$ and $\mathbf{4 d}$ exhibited efficient activities against $C$. albicans with $\mathrm{MIC}$ values ranging from 0.047 to $0.095 \mathrm{mM}$. It was specially noted that deprotected glucose-derived bis-1,2,3-triazole $\mathbf{8 a}$ bearing C-8 alkyl chain moiety gave excellent inhibitory activity against $A$. fumigatus with an MIC value of $0.055 \mathrm{mM}$ compared to Fluconazole.

From the antifungal data in Table 2, glucose derived bis-1,2,3triazole 5d showed no obvious antifungal activity, but the corresponding hydrochloride $\mathbf{4 d}$ with better water solubility exhibited enhanced activity with an MIC of $0.047 \mathrm{mM}$ superior to the reference drug Fluconazole. Introduction of chloro substituent in C-4 position of benzyl triazole 5d, which afforded 3,4dichloro substituted benzyl triazole 5c, also showed no inhibitory potency against $C$. albicans. But its hydrochloride $4 \mathbf{c}$ showed the decrease of inhibitory efficiency against $C$. albicans with the MIC value of $0.086 \mathrm{mM}$ in contrast to the hydrochloride of monochlorophenyl compound $4 \mathbf{d}$. Besides, compound $4 \mathbf{e}$ with chloro at para position of benzyl group only gave moderate antifungal activity against $C$. albicans and $A$. fumigatus. While introduction of chloro substituent in C-2 position of the predecessor of compound $\mathbf{5 e}$, which yielded 2,4-dichloro substituted benzyl 1,2,3-triazole $\mathbf{5 b}$, showed good inhibitory potency against $C$. albicans. This suggested significant effect of the water-solubility of compounds and substitution in benzyl group on antifungal activities.

Antifungal assay also revealed that the alkyl chain length in the triazole ring had noticeable effect on the antimicrobial activities. Alkyl 1,2,3-triazole 8a with $\left(\mathrm{CH}_{2}\right)_{8}$ chain gave fourteen times higher potency than Fluconazole against $A$. fumigatus, and showed moderate inhibitory activity against $C$. albicans with an MIC value of $0.22 \mathrm{mM}$. The corresponding hydrochloride $7 \mathbf{a}$ also gave moderate antifungal activity against $C$. albicans and A. fumigatus with MIC values of 0.39 and $0.20 \mathrm{mM}$ respectively. While alkyl 1,2,3-triazoles $\mathbf{7 b}$ and $\mathbf{8 b}$ carrying $\left(\mathrm{CH}_{2}\right)_{12}$ moiety gave no obvious inhibiton against tested fungal strains. Therefore, the introduction of linear alkyl chain with proper lengths into 1,2,3-triazole ring could enhance antifungal activities. Further study in vivo of these bioactive compounds as antifungal agents is in progress.

Antibacterial activity: The antibacterial evaluation revealed that for almost bio-active glucose derived benzyl 1,2,3-triazoles, the hydrochlorides in general showed better antibacterial activity than their corresponding precursors. It's worth mentioning that compounds $\mathbf{8 a}, \mathbf{4 c}$ and $\mathbf{4 d}$ displayed the most prominent activities towards tested bacterial strains.

Based on the antibacterial data in Table 2, it was observed that bis-triazole 8 a bearing C- 8 alkyl chain showed the strongest and broad-spectrum antibacterial activity among all the synthesized compounds. Compound $\mathbf{8 a}$ gave significant inhibiton against $S$. aureus, $P$. aeruginosa and $B$. proteus with MIC value of $0.055 \mathrm{mM}$ comparable or superior to Chloramphenicol, while its corresponding hydrochloride $7 \mathbf{a}$ gave better antibacterial activity against P.aeruginos $a$ and $E$. coli than its predecessor. Conversely, the antibacterial results showed no obvious inhibition for compounds $\mathbf{7 b}$ and $\mathbf{8 b}$ with long alkyl chain. Therefore, the introduction of linear alkyl with $\left(\mathrm{CH}_{2}\right)_{8}$ alkyl chain into 1,2,3-triazole ring may be more useful to enhance antibacterial activities.

Among the substituted benzyl 1,2,3-triazoles, hydrochloride 4c containing 3,4-dichlorobenzyl group, which showed more potent antibacterial activity than compound $\mathbf{4 d}$ with 3 -chlorobenzyl group, could significantly inhibit the growth of $E$. coli at concentration of $0.043 \mathrm{mM}$. Compound $4 \mathbf{c}$ also exerted moderate inhibitory activities against $B$. subtilis and $B$. proteus with MIC value of $0.34 \mathrm{mM}$ compared to the reference drug Chloramphenicol. The hydrochloride $4 \mathbf{e}$ bearing chloro substituent in para position of benzene exhibited weaker antibacterial activity against $B$. subtilis, $B$. proteus and $E$. coli than compound $\mathbf{4 c}$. Bis-triazole $\mathbf{5 b}$ with 2,4-dichloro moiety showed moderate inhibitory potency against $P$. aeruginosa, B. subtilis and E. coli. In vitro antibacterial tests demonstrated that water solubility of the synthesized compounds, the structural factors such as the substituents in benzene ring and the length of alkyl chain could largely affect their antibacterial activities.

\section{Conclusion}

In summary, a series of novel glucose derived bis-1,2,3-triazoles have been synthesized in excellent yields via $\mathrm{Cu}(\mathrm{I})$ catalyzed 1,3-dipolar cycloaddition. Antimicrobial activity was evaluated. The results obtained from this study suggested that the water solubility of compounds, substituents in benzene ring and alkyl chain length in 1,2,3-triazole ring had significant effect on the antimicrobial activities. Among their deprotected derivatives, compound $\mathbf{8 a}$ with $\left(\mathrm{CH}_{2}\right)_{8}$ alkyl chain gave fourteen times higher potency than Fluconazole against $A$. fumigatus. It also showed the broad and good antibacterial activity. These results showed that the present series containing 1,2,3-triazole as a lead are necessary to further investigate for new antimicrobial agents with high activity and low toxicity.

\section{Experimental}

Chemistry. All air- and moisture sensitive reactions were carried out in flame dried, $\mathrm{N}_{2}$-flushed, double-neck round bottom flask sealed with rubber septa, and the reagents were injected with a syringe. Tetrahydrofuran (THF) was freshly distilled from sodium-benzophenone. Methyl 4,6-O-benzylidene $\alpha$-D-glucopyranoside was prepared from methyl $\alpha$-D-glucopyraoside (1). 
All other chemicals and reagents were obtained from commercial suppliers and used without further purification. TLC analyses were done using pre-coated silica gel plates and visualization was done using UV lamp at $254 \mathrm{~nm}$ and $\mathrm{I}_{2} .{ }^{1} \mathrm{H}$ and ${ }^{13} \mathrm{C}$ NMR spectra were recorded on Bruker AV-300 or Varian-Mercury 400 spectrometer instrument (Bruker Bioscience, Varian, Palo Alto, CA, respectively, both USA). The chemical shifts were given with respect to tetramethylsilane $\left({ }^{1} \mathrm{H}: \delta 0 \mathrm{ppm}\right)$ and $\mathrm{CDCl}_{3}\left({ }^{13} \mathrm{C}: \delta 77.0 \mathrm{ppm}\right)$. Coupling constants were determined directly from ${ }^{1} \mathrm{H}$ NMR spectra. The mass spectra were recorded on FINIGAN TRACE GC/MS (Thermo Electron Corporation, Bremen, Germany). FT-IR spectra were carried out on Bruker RFS100/S spectrophotometer (Bio-Rad, Cambridge, MA, USA) using $\mathrm{KBr}$ pellets in the $400-4000 \mathrm{~cm}^{-1}$ range. Melting points were recorded on X-6 melting point apparatus.

Methyl 4,6-O-benzylidene-2,3-di-O-propargyl-o-D-glucopyranoside (2). To a solution of $5.0 \mathrm{~g}(17.70 \mathrm{mmol})$ methyl 4,6-O-benzylidene $\alpha$-D-glucopyranoside in $80 \mathrm{~mL}$ anhydrous THF was added $3.3 \mathrm{~g}(0.14 \mathrm{~mol})$ sodium hydride. The suspension was stirred for 45 minutes and cooled with an ice-salt bath. With vigorous stirring, $4.8 \mathrm{~mL}$ of propargyl bromide was added slowly, and the reaction mixture was stirred for 2 days at room temperature. Brash ice was added carefully until no gas was generated. The solvent was removed under reduced pressure and the residue was extracted with ethyl acetate $(40 \mathrm{~mL} \times 3)$. The combined ethyl acetate solutions were washed with saturated $\mathrm{NaCl}$ solution, dried with anhydrous sodium sulfate and then concentrated under reduced pressure. The residue was subjected to column chromatography on flash silica gel using ethyl acetate/petroleum $(1 / 4, \mathrm{v} / \mathrm{v})$ as eluent to give methyl 4,6-Obenzylidene-2,3-di-O-propargyl- $\alpha$-D-glucopyranoside (2) as white solid (5.14 g, 81\%). mp $75-77^{\circ} \mathrm{C}$ (lit. ${ }^{31} \mathrm{mp} 78-79^{\circ} \mathrm{C}$ ). ${ }^{1} \mathrm{H}$ NMR $\left(300 \mathrm{MHz}, \mathrm{CDCl}_{3}, J\right.$ in $\left.\mathrm{Hz}\right) \delta$ 7.42-7.40 (m, 2H, Ph 3,5-H), 7.31-7.29 (m, 3H, Ph 2,4,6-H), 5.47 (s, 1H, Ph CH), $4.81\left(\mathrm{~d}, 1 \mathrm{H},{ }^{3} J_{1,2}=3.7,1-\mathrm{H}\right), 4.40-4.35\left(\mathrm{~m}, 4 \mathrm{H}, 2 \equiv \mathrm{CCH}_{2}\right), 4.21$ $\left(\mathrm{dd}, 1 \mathrm{H},{ }^{3} J_{6 \mathrm{eq}, 5}=4.1,{ }^{2} J_{6 \mathrm{eq}, 6 \mathrm{ax}}=9.6,6-\mathrm{H}_{\mathrm{eq}}\right), 3.93\left(\mathrm{t}, 1 \mathrm{H},{ }^{3} J_{3,2}=\right.$ $\left.{ }^{3} J_{3,4}=9.2,3-\mathrm{H}\right), 3.67$ (ddd, $1 \mathrm{H},{ }^{3} J_{5,6 \mathrm{eq}}=4.1,{ }^{3} J_{5,4}=9.6,{ }^{3} J_{5,6 \mathrm{ax}}=$ $10,5-\mathrm{H}), 3.66-3.62\left(\mathrm{~m}, 2 \mathrm{H}, 2-\mathrm{H}, 6-\mathrm{H}_{\mathrm{ax}}\right), 3.55\left(\mathrm{t}, 1 \mathrm{H},{ }^{3} J_{4,3}=9.2\right.$, $\left.{ }^{3} J_{4,5}=9.6,4-\mathrm{H}\right), 3.38\left(\mathrm{~s}, 3 \mathrm{H}, \mathrm{OCH}_{3}\right), 2.39-2.36(\mathrm{~m}, 1 \mathrm{H}, \equiv \mathrm{CH})$, $2.10(\mathrm{~s}, 1 \mathrm{H}, \equiv \mathrm{CH}) ;{ }^{13} \mathrm{C} \mathrm{NMR}\left(75 \mathrm{MHz}, \mathrm{CDCl}_{3}\right) \delta 137.20,128.94$, 128.19, 125.96, 101.29, 99.22, 81.94, 79.92, 79.89, 77.86, 74.85, 74.36, 68.96, 62.07, 59.97, 59.34, 55.23.

General procedure for the $\mathbf{C u}(\mathrm{I})$-catalyzed synthesis of glucose derived 1,2,3-triazoles 3a-i and 6a-b. To a solution of 800 $\mathrm{mg}(2.23 \mathrm{mmol})$ of compound $\mathbf{2}$ in $20 \mathrm{~mL}$ of a DMSO-water mixture $(4 / 1, \mathrm{v} / \mathrm{v})$ was added $1.0 \mathrm{~g}(5.97 \mathrm{mmol})$ of benzyl or alkyl azide. Hereafter $93 \mathrm{mg}(0.45 \mathrm{mmol})$ sodium ascorbate and $63 \mathrm{mg}(0.22 \mathrm{mmol})$ of copper (II) sulfate pentahydrate was added successively, and the mixture was stirred for 2 days at room temperature. The reaction mixture was diluted with $40 \mathrm{~mL}$ of cooled water and cooled with a salt-ice bath: a white precipitate was formed, filtrated and washed three times with $20 \mathrm{~mL}$ portions of cold water. The moist solid was extracted in $20 \mathrm{~mL}$ of $\mathrm{CHCl}_{3}$ and $10 \mathrm{~mL}$ of water. After phase separation, the organic phase was carefully washed with saturated sodium chloride solution and dried with anhydrous sodium sulfate, then concentrated under reduced pressure. The residue was subjected to column chromatography on silica using ethyl acetate/petrol- eum ether $(5 / 1, \mathrm{v} / \mathrm{v})$ as eluent to give compounds $\mathbf{3 a - i}$ and $\mathbf{6 a - b}$.

4,4'-((6R,7R,8R,8aS)-6-Methoxy-2-phenyl-hexahydropyrano[3,2- $d][1,3]$ dioxine-7,8-diyl)bis(oxy)bis(methylene) bis(1-(2-chlorobenzyl)-1H-1,2,3-triazole) (3a): Ruddy solid. mp 94 - $95{ }^{\circ} \mathrm{C}$. Yield: $68 \%$. ${ }^{1} \mathrm{H}$ NMR $\left(400 \mathrm{MHz} \mathrm{CDCl}_{3}, J\right.$ in $\mathrm{Hz}) \delta 7.75(\mathrm{~s}, 1 \mathrm{H}$, triazole $\mathrm{H}), 7.61(\mathrm{~s}, 1 \mathrm{H}$, triazole $\mathrm{H}), 7.45-7.42$ (m, 2H, Ar H), 7.40 (dd, $\left.1 \mathrm{H},{ }^{4} J=1.6,{ }^{3} J=7.6, \operatorname{Ar~H}\right), 7.36$ (dd, $1 \mathrm{H},{ }^{4} J=1.6,{ }^{3} \mathrm{~J}=7.6$, Ar H), 7.34-7.31 (m, 3H, Ar H), 7.307.28 (m, 1H, Ar H), 7.25-7.21 (m, 2H, Ar H), 7.19-7.15 (m, $2 \mathrm{H}, \mathrm{Ar} \mathrm{H}), 6.98\left(\mathrm{dd}, 1 \mathrm{H},{ }^{4} J=1.2,{ }^{3} J=7.6, \mathrm{Ar} \mathrm{H}\right), 5.61(\mathrm{~d}, 2 \mathrm{H}$, $\left.{ }^{4} J=1.6, \mathrm{OCH}_{2}\right), 5.50(\mathrm{~s}, 1 \mathrm{H}, \mathrm{Ph}-\mathrm{CH}), 5.47\left(\mathrm{~s}, 2 \mathrm{H}, \mathrm{OCH}_{2}\right)$, 4.99-4.89 (m, 2H, $\left.\mathrm{NCH}_{2}\right), 4.87-4.80\left(\mathrm{~m}, 2 \mathrm{H}, \mathrm{NCH}_{2}\right), 4.78$ (d, $\left.1 \mathrm{H},{ }^{3} J_{1,2}=3.6,1-\mathrm{H}\right), 4.25\left(\mathrm{dd}, 1 \mathrm{H},{ }^{3} J_{6 \mathrm{eq}, 5}=4.8,{ }^{2} J_{6 \mathrm{eq}, 6 \mathrm{ax}}=9.6\right.$, $\left.6-\mathrm{H}_{\mathrm{eq}}\right), 3.93\left(\mathrm{t}, 1 \mathrm{H},{ }^{3} J_{4,3}=9.2,{ }^{3} J_{4,5}=9.6,3-\mathrm{H}\right), 3.78(\mathrm{ddd}, 1 \mathrm{H}$, $\left.{ }^{3} J_{5,6 \mathrm{eq}}=4.8,{ }^{3} J_{5,4}=9.6,{ }^{3} J_{5,6 \mathrm{ax}}=10,5-\mathrm{H}\right), 3.69\left(\mathrm{t}, 1 \mathrm{H},{ }^{3} J_{6 \mathrm{ax}, 5}=\right.$ $\left.10.0,6-\mathrm{H}_{\mathrm{ax}}\right), 3.61\left(\mathrm{dd}, 1 \mathrm{H},{ }^{3} J_{2,1}=4.0,{ }^{3} J_{2,3}=9.2,2-\mathrm{H}\right), 3.56(\mathrm{t}$, $\left.1 \mathrm{H},{ }^{3} J_{4,3}=9.2,{ }^{3} J_{4,5}=9.6,4-\mathrm{H}\right), 3.35\left(\mathrm{~s}, 3 \mathrm{H}, \mathrm{OCH}_{3}\right) ;{ }^{13} \mathrm{C} \mathrm{NMR}$ $\left(75 \mathrm{MHz}, \mathrm{CDCl}_{3}\right) \delta 145.64,144.90,137.12,134.34,133.35$, 133.04, 132.48, 132.28, 130.26, 130.06, 129.83, 129.74, 129.58, 128.92, 128.11, 127.41, 127.34, 125.94, 123.61, 123.57, 123.20, 101.32, 98.50, 81.57, 79.21, 78.11, 68.85, 66.22, 64.64, 62.11, 55.08, 51.27, 51.06; IR (KBr) v 3138, 2924, 2856, 2598, 2363, 1600, 1577, 1457, 1435, 1408, 1321, 1300, 1224, 1169, 1069, $1004 \mathrm{~cm}^{-1}$; MS $(\mathrm{m} / z): 715[\mathrm{M}+\mathrm{Na}]^{+}, 683\left[\mathrm{M}+\mathrm{Na}-\mathrm{CH}_{3} \mathrm{OH}\right]^{+}$, $627[\mathrm{M}+\mathrm{K}-\mathrm{PhCHO}]^{+}, 595\left[\mathrm{M}+\mathrm{K}-\mathrm{PhCHO}-\mathrm{CH}_{3} \mathrm{OH}\right]^{+}$.

4,4'-((6R,7R,8R,8aS)-6-Methoxy-2-phenyl-hexahydropyrano[3,2-d][1,3]dioxine-7,8-diyl)bis(oxy)bis(methylene) bis(1-(2,4-dichlorobenzyl)-1H-1,2,3-triazole) (3b): White solid. mp 168 - $170{ }^{\circ} \mathrm{C}$. Yield: $92 \% .{ }^{1} \mathrm{H}$ NMR $\left(400 \mathrm{MHz}, \mathrm{CDCl}_{3}, J\right.$ in $\mathrm{Hz}) \delta 7.81(\mathrm{~s}, 1 \mathrm{H}$, triazole $\mathrm{H}), 7.63(\mathrm{~s}, 1 \mathrm{H}$, triazole $\mathrm{H}), 7.46-7.43$ (m, 3H, Ar H), 7.39 (d, $\left.1 \mathrm{H},{ }^{4} J=2.0, \operatorname{Ar~H}\right), 7.35-7.33(\mathrm{~m}, 3 \mathrm{H}$, Ar H), 7.22 (dd, $\left.1 \mathrm{H},{ }^{4} J=2.0,{ }^{3} J=8.4, \operatorname{ArH}\right), 7.15\left(\mathrm{dd}, 1 \mathrm{H},{ }^{4} \mathrm{~J}=\right.$ $\left.2.0,{ }^{3} J=8.4, \operatorname{Ar~H}\right), 7.12\left(\mathrm{~d}, 1 \mathrm{H},{ }^{3} J=8.4, \mathrm{Ar} \mathrm{H}\right), 6.90(\mathrm{~d}, 1 \mathrm{H}$, $\left.{ }^{3} J=8.0, \mathrm{Ar} \mathrm{H}\right), 5.59\left(\mathrm{~s}, 2 \mathrm{H}, \mathrm{OCH}_{2}\right), 5.51(\mathrm{~s}, 1 \mathrm{H}, \mathrm{Ph}-\mathrm{CH}), 5.44$ (s, 2H, $\left.\mathrm{OCH}_{2}\right), 4.99-4.89\left(\mathrm{~m}, 2 \mathrm{H}, \mathrm{NCH}_{2}\right), 4.84\left(\mathrm{~s}, 2 \mathrm{H}, \mathrm{NCH}_{2}\right)$, $4.80\left(\mathrm{~d}, 1 \mathrm{H},{ }^{3} J_{1,2}=3.6,1-\mathrm{H}\right), 4.26\left(\mathrm{dd}, 1 \mathrm{H},{ }^{3} J_{6 \mathrm{eq}, 5}=4.4,{ }^{2} J_{6 \mathrm{eq}, 6 \mathrm{ax}}=\right.$ $10.0,6-\mathrm{H}_{\text {eq }}$ ), $3.93\left(\mathrm{t}, 1 \mathrm{H},{ }^{3} J_{3,4}={ }^{3} J_{3,2}=9.2,3-\mathrm{H}\right), 3.79$ (ddd, $\left.1 \mathrm{H},{ }^{3} J_{5,6 \mathrm{eq}}=4.4,{ }^{3} J_{5,4}=9.2,{ }^{3} J_{5,6 \mathrm{ax}}=10,5-\mathrm{H}\right), 3.70\left(\mathrm{t}, 1 \mathrm{H},{ }^{3} J_{6 \mathrm{ax}, 5}=\right.$ $\left.{ }^{2} J_{6 \mathrm{ax}, 6 \mathrm{eq}}=10,6-\mathrm{H}_{\mathrm{ax}}\right), 3.60\left(\mathrm{dd}, 1 \mathrm{H},{ }^{3} J_{2,1}=3.6,{ }^{3} J_{2,3}=9.2,2-\mathrm{H}\right)$, $3.56\left(\mathrm{t}, 1 \mathrm{H},{ }^{3} J_{4,3}={ }^{3} J_{4,5}=9.2,4-\mathrm{H}\right), 3.38\left(\mathrm{~s}, 3 \mathrm{H}, \mathrm{OCH}_{3}\right) ;{ }^{13} \mathrm{C}$ NMR $\left(100 \mathrm{MHz}, \mathrm{CDCl}_{3}\right) \delta 145.90,145.19,137.28,135.46$, 135.22, 134.17, 133.82, 131.31, 131.18, 131.13, 130.67, 129.70, $129.54,129.05,128.22,127.85,127.79,126.07,123.63,123.26$, 101.48, 98.62, 81.79, 79.37, 78.15, 69.01, 66.36, 64.86, 62.26, 55.22, 50.75, 50.57; IR (KBr) v 3130, 3093, 2925, 2876, 2361, 2339, 1619, 1592, 1563, 1475, 1455, 1384, 1332, 1218, 1132, $1110,1090 \mathrm{~cm}^{-1}$; MS $(\mathrm{m} / \mathrm{z}): 785[\mathrm{M}+\mathrm{Na}]^{+}$.

4,4'-((6R,7R,8R,8aS)-6-Methoxy-2-phenyl-hexahydropyrano[3,2- $d][1,3]$ dioxine-7,8-diyl)bis(oxy)bis(methylene) bis(1-(3,4-dichlorobenzyl)-1H-1,2,3-triazole) (3c): White solid. mp 102 - $104{ }^{\circ} \mathrm{C}$. Yield: $75 \%$. ${ }^{1} \mathrm{H}$ NMR (400 MHz, $\mathrm{CDCl}_{3}, J$ in $\mathrm{Hz}) \delta 7.86(\mathrm{~s}, 1 \mathrm{H}$, triazole $\mathrm{H}), 7.61(\mathrm{~s}, 1 \mathrm{H}$, triazole $\mathrm{H}), 7.47-7.45$ (m, 2H, Ar H), 7.39 (d, $\left.1 \mathrm{H},{ }^{3} J=8.4, \operatorname{Ar~H}\right), 7.37-7.34(\mathrm{~m}, 5 \mathrm{H}$, Ar H), $7.25\left(\mathrm{~d}, 1 \mathrm{H},{ }^{4} \mathrm{~J}=2.0, \mathrm{ArH}\right), 7.13\left(\mathrm{dd}, 1 \mathrm{H},{ }^{4} \mathrm{~J}=2.0,{ }^{3} \mathrm{~J}=\right.$ 8.0, Ar H), $6.96\left(\mathrm{dd}, 1 \mathrm{H},{ }^{4} J=2.4,{ }^{3} J=8.0, \mathrm{Ar} \mathrm{H}\right), 5.50(\mathrm{~s}, 1 \mathrm{H}$, $\mathrm{Ph}-\mathrm{CH}), 5.49-5.40\left(\mathrm{~m}, 2 \mathrm{H}, \mathrm{OCH}_{2}\right), 5.32-5.23\left(\mathrm{~m}, 2 \mathrm{H}, \mathrm{OCH}_{2}\right)$, 4.95-4.86 (m, $\left.2 \mathrm{H}, \mathrm{NCH}_{2}\right), 4.85-4.77\left(\mathrm{~m}, 3 \mathrm{H}, \mathrm{NCH}_{2}, 1-\mathrm{H}\right), 4.26$ $\left(\mathrm{dd}, 1 \mathrm{H},{ }^{3} J_{6 \mathrm{eq}, 5}=4.8,{ }^{2} J_{6 \mathrm{eq}, 6 \mathrm{ax}}=10,6-\mathrm{H}_{\mathrm{eq}}\right), 3.90\left(\mathrm{t}, 1 \mathrm{H},{ }^{3} J_{3,4}=\right.$ 
$\left.{ }^{3} J_{3,2}=9.2,3-\mathrm{H}\right), 3.78\left(\mathrm{ddd}, 1 \mathrm{H},{ }^{3} J_{5,6 \mathrm{eq}}=4.8,{ }^{3} J_{5,4}=9.2,{ }^{3} J_{5,6 \mathrm{ax}}=\right.$ $9.6,5-\mathrm{H}), 3.70\left(\mathrm{t}, 1 \mathrm{H},{ }^{2} J_{6 \mathrm{ax}, 6 \mathrm{eq}}=10,6-\mathrm{H}_{\mathrm{ax}}\right), 3.57\left(\mathrm{dd}, 1 \mathrm{H},{ }^{3} J_{2,1}=\right.$ $\left.3.6,{ }^{3} J_{2,3}=9.2,2-\mathrm{H}\right), 3.54\left(\mathrm{t}, 1 \mathrm{H},{ }^{3} J_{4,3}={ }^{3} J_{4,5}=9.2,4-\mathrm{H}\right), 3.38$ $\left(\mathrm{s}, 3 \mathrm{H}, \mathrm{OCH}_{3}\right) ;{ }^{13} \mathrm{C} \mathrm{NMR}\left(100 \mathrm{MHz}, \mathrm{CDCl}_{3}\right) \delta 146.06,145.38$, 137.32, 134.87, 134.85, 133.09, 133.02, 132.92, 132.84, 130.94, $130.91,129.97,129.77,129.03,128.22,127.35,127.11,126.10$, $123.51,123.03,101.47,98.42,81.76,79.32,78.03,69.96,66.25$, 64.68, 62.22, 55.20, 52.70, 52.51; IR (KBr) v 3134, 3094, 2994, 2920, 2875, 2361, 2060, 1639, 1563, 1473, 1453, 1403, 1368, 1334, 1283, 1214, 1192, 1114, 1089, 1051, 1032, 1007 $\mathrm{cm}^{-1}$; EI-MS $m / z 785[\mathrm{M}+\mathrm{Na}]^{+}, 763[\mathrm{M}+\mathrm{H}]^{+}$.

4,4'-((6R,7R,8R,8aS)-6-Methoxy-2-phenyl-hexahydropyrano $[3,2-d][1,3]$ dioxine-7,8-diyl)bis(oxy)bis(methylene) bis(1-(3-chlorobenzyl)-1H-1,2,3-triazole) (3d): Syrup. Yield: $74 \%$. ${ }^{1} \mathrm{H} \mathrm{NMR}\left(300 \mathrm{MHz} \mathrm{CDCl}_{3}, J\right.$ in $\left.\mathrm{Hz}\right) \delta 7.74(\mathrm{~s}, 1 \mathrm{H}$, triazole $\mathrm{H}), 7.52(\mathrm{~s}, 1 \mathrm{H}$, triazole $\mathrm{H}), 7.39-7.38(\mathrm{~m}, 2 \mathrm{H}, \mathrm{Ar} \mathrm{H}), 7.29-7.27$ (m, 3H, Ar H), 7.22-7.15 (m, 5H, Ar H), 7.12-7.08 (m, 2H, Ar H), $6.95\left(\mathrm{~d}, 1 \mathrm{H},{ }^{3} J=7.0, \mathrm{Ar} \mathrm{H}\right), 5.44(\mathrm{~s}, 1 \mathrm{H}, \mathrm{Ph}-\mathrm{CH}), 5.39$ (d, $\left.2 \mathrm{H},{ }^{3} \mathrm{~J}=5.3, \mathrm{OCH}_{2}\right), 5.23\left(\mathrm{~s}, 2 \mathrm{H}, \mathrm{OCH}_{2}\right), 4.90-4.79(\mathrm{~m}, 2 \mathrm{H}$, $\left.\mathrm{NCH}_{2}\right), 4.75\left(\mathrm{~s}, 3 \mathrm{H}, \mathrm{NCH}_{2}, 1-\mathrm{H}\right), 4.19\left(\mathrm{dd}, 1 \mathrm{H},{ }^{3} J_{6 \mathrm{eq}, 5}=4.0\right.$, $\left.{ }^{2} J_{6 \mathrm{eq}, 6 \mathrm{ax}}=9.5,6-\mathrm{H}_{\mathrm{eq}}\right), 3.84\left(\mathrm{t}, 1 \mathrm{H},{ }^{3} J_{3,4}={ }^{3} J_{3,2}=9.2,3-\mathrm{H}\right), 3.73$ (ddd, $\left.1 \mathrm{H},{ }^{3} J_{5,6 \mathrm{eq}}=4.4,{ }^{3} J_{5,4}=9.6,{ }^{3} J_{5,6 \mathrm{ax}}=10,5-\mathrm{H}\right), 3.62(\mathrm{t}, 1 \mathrm{H}$, $\left.{ }^{3} J_{6 \mathrm{ax}, 5}={ }^{2} J_{6 \mathrm{ax}, 6 \mathrm{eq}}=9.8,6-\mathrm{H}_{\mathrm{ax}}\right), 3.54-3.45(\mathrm{~m}, 2 \mathrm{H}, 2-\mathrm{H}, 4-\mathrm{H}), 3.30$ $\left(\mathrm{s}, 3 \mathrm{H}, \mathrm{OCH}_{3}\right) ;{ }^{13} \mathrm{C} \mathrm{NMR}\left(75 \mathrm{MHz}, \mathrm{CDCl}_{3}\right) \delta 145.99,145.27$, 137.27, 136.63, 130.29, 130.24, 129.05, 128.95, 128.82, 128.73, $128.26,128.12$, 127.95, 126.20, 126.09, 125.98, 123.54, 122.98, $101.45,98.42,81.70,79.25,78.12,68.96,66.35,64.74,62.19$, 55.20, 53.31, 53.12; IR (KBr) v 3123, 3074, 29664, 2918, 2609, 2362, 1620, 1574, 1475, 1447, 1401, 1370, 1327, 1281, 1221, $1196,1089,1051,1003 \mathrm{~cm}^{-1}$; MS $(\mathrm{m} / \mathrm{z}): 715[\mathrm{M}+\mathrm{Na}]^{+}, 693[\mathrm{M}]^{+}$.

4,4'-((6R,7R,8R,8aS)-6-Methoxy-2-phenyl-hexahydropyrano $[3,2-d][1,3]$ dioxine-7,8-diyl)bis(oxy)bis(methylene) bis(1-(4-chlorobenzyl)-1H-1,2,3-triazole) (3e): White solid. mp $152-153{ }^{\circ} \mathrm{C}$. Yield: $75 \%$. ${ }^{1} \mathrm{H}$ NMR $\left(300 \mathrm{MHz}, \mathrm{CDCl}_{3}, J\right.$ in $\mathrm{Hz}) \delta 7.76(\mathrm{~s}, 1 \mathrm{H}$, triazole $\mathrm{H}), 7.57(\mathrm{~s}, 1 \mathrm{H}$, triazole $\mathrm{H}), 7.47-7.45$ (m, 2H, Ar H), 7.36-7.33 (m, 4H, Ar H), 7.30 (s, 1H, Ar H), 7.27-7.22 (m, 4H, Ar H), 7.07 (d, 2H, ${ }^{3} J=8.2$, Ar H), 5.51 (s, $1 \mathrm{H}, \mathrm{Ph}-\mathrm{CH}), 5.46-5.40\left(\mathrm{~m}, 2 \mathrm{H}, \mathrm{OCH}_{2}\right), 5.31\left(\mathrm{~s}, 2 \mathrm{H}, \mathrm{OCH}_{2}\right)$, 4.97-4.86 (m, 2H, $\left.\mathrm{NCH}_{2}\right), 4.81$ (s, 3H, $\left.\mathrm{NCH}_{2}, 1-\mathrm{H}\right), 4.26$ (dd, $\left.1 \mathrm{H},{ }^{3} J_{6 \mathrm{eq}, 5}=4.0,{ }^{2} J_{6 \mathrm{eq}, 6 \mathrm{ax}}=9.5,6-\mathrm{H}_{\mathrm{eq}}\right), 3.91\left(\mathrm{t}, 1 \mathrm{H},{ }^{3} J_{3,4}={ }^{3} J_{3,2}=\right.$ $9.2,3-\mathrm{H}), 3.80\left(\mathrm{ddd}, 1 \mathrm{H},{ }^{3} J_{5,6 \mathrm{eq}}=4.0,{ }^{3} J_{5,4}=9.6,{ }^{3} J_{5,6 \mathrm{ax}}=10,5-\mathrm{H}\right)$, $3.70\left(\mathrm{t}, 1 \mathrm{H},{ }^{3} J_{6 \mathrm{ax}, 5}=10,{ }^{3} J_{6 \mathrm{ax}, 6 \mathrm{eq}}=9.7,6-\mathrm{H}_{\mathrm{ax}}\right), 3.60\left(\mathrm{~d}, 1 \mathrm{H},{ }^{3} J_{2,1}=\right.$ $3.5,2-\mathrm{H}), 3.55\left(\mathrm{t}, 1 \mathrm{H},{ }^{3} J_{4,3}=9.1,{ }^{3} J_{4,5}=9.1,4-\mathrm{H}\right), 3.35(\mathrm{~s}, 3 \mathrm{H}$, $\left.\mathrm{OCH}_{3}\right) ;{ }^{13} \mathrm{C} \mathrm{NMR}\left(75 \mathrm{MHz}, \mathrm{CDCl}_{3}\right) \delta 145.90,145.19,137.22$, $134.56,134.43,133.16,129.46,129.21,129.12,129.05,128.20$, $126.03,123.28,122.80,101.36,98.39,81.65,79.24,78.05$, $68.90,66.34,64.76,62.13,55.14,53.21,53.02$; IR (KBr) v 3135, 3083, 2986, 2915, 2858, 1588, 1493, 1461, 1451, 1411, 1366, 1320, 1224, 1181, 1133, 1110, 1090, $1052 \mathrm{~cm}^{-1}$; MS (m/z): 691 $[\mathrm{M}+\mathrm{H}]^{+}$.

4,4'-((6R,7R,8R,8aS)-6-Methoxy-2-phenyl-hexahydropyrano $[3,2-d][1,3]$ dioxine-7,8-diyl)bis(oxy)bis(methylene) bis(1-(2,4-difluorobenzyl)-1H-1,2,3-triazole) (3f): White solid. mp 162-163 ${ }^{\circ} \mathrm{C}$. Yield: $92 \%$. ${ }^{1} \mathrm{H}$ NMR (400 MHz, $\mathrm{CDCl}_{3}, J$ in $\mathrm{Hz}) \delta 7.78(\mathrm{~s}, 1 \mathrm{H}$, triazole $\mathrm{H}), 7.62(\mathrm{~s}, 1 \mathrm{H}$, triazole $\mathrm{H}), 7.48-7.44$ (m, 2H, Ar H), 7.38-7.34 (m, 3H, Ar H), 7.33-7.28 (m, 1H, Ar H), 7.17-7.12 (m, 1H, Ar H), 6.90-6.85 (m, 2H, Ar H), 6.84-6.79 (m, 2H, Ar H), 5.52 (s, 3H, $\left.\mathrm{Ph} \mathrm{CH}, \mathrm{OCH}_{2}\right), 5.39$ (s, 2H, $\left.\mathrm{OCH}_{2}\right)$,
4.97-4.88 (m, 2H, $\left.\mathrm{NCH}_{2}\right), 4.83\left(\mathrm{~d}, 2 \mathrm{H},{ }^{4} \mathrm{~J}=2.0, \mathrm{NCH}_{2}\right), 4.80$ $\left(\mathrm{d}, 1 \mathrm{H},{ }^{3} J_{1,2}=3.6,1-\mathrm{H}\right), 4.27\left(\mathrm{dd}, 1 \mathrm{H},{ }^{3} J_{6 \mathrm{eq}, 5}=4.4,{ }^{2} J_{6 \mathrm{eq}, 6 \mathrm{ax}}=9.6\right.$, $\left.6-\mathrm{H}_{\mathrm{eq}}\right), 3.93\left(\mathrm{t}, 1 \mathrm{H},{ }^{3} J_{4,3}=9.2,{ }^{3} J_{4,5}=9.6,3-\mathrm{H}\right), 3.80(\mathrm{ddd}, 1 \mathrm{H}$, $\left.{ }^{3} J_{5,6 \mathrm{eq}}=4.4,{ }^{3} J_{5,4}=9.6,{ }^{3} J_{5,6 \mathrm{ax}}=10,5-\mathrm{H}\right), 3.72\left(\mathrm{t}, 1 \mathrm{H},{ }^{3} J_{6 \mathrm{ax}, 5}=\right.$ $\left.10,6-\mathrm{H}_{\mathrm{ax}}\right), 3.60\left(\mathrm{dd}, 1 \mathrm{H},{ }^{3} J_{2,1}=3.6,{ }^{3} J_{2,3}=9.2,2-\mathrm{H}\right), 3.56(\mathrm{t}, 1 \mathrm{H}$, $\left.{ }^{3} J_{4,3}={ }^{3} J_{4,5}=9.2,4-\mathrm{H}\right), 3.37\left(\mathrm{~s}, 3 \mathrm{H}, \mathrm{OCH}_{3}\right) ;{ }^{13} \mathrm{C} \mathrm{NMR}(100 \mathrm{MHz}$, $\left.\mathrm{CDCl}_{3}\right) \delta 164.63,164.51,164.39,162.13,162.01,161.95$, $161.89,161.78,159.58,159.40,146.02,145.35,137.38,131.78$, $131.73,131.68,131.46,131.41,131.36,131.31,129.02,128.24$, $126.11,123.31,122.90,118.30,118.22,118.19,118.07,118.03$, $112.19,112.16,112.07,111.98,111.94,111.89,111.85,104.59$, $104.47,104.34,104.22,104.09,103.96,101.52,98.70,81.87$, $79.48,78.30,69.06,66.45,64.93,62.30,55.22,47.08,47.04$, 46.90, 46.87; IR (KBr) v 3122, 3079, 2920, 2879, 1620, 1606, 1559, 1510, 1456, 1432, 1369 1332, 1276, 1144, 1091, 1050, $1006 \mathrm{~cm}^{-1}$; MS $(\mathrm{m} / \mathrm{z}): 697[\mathrm{M}+\mathrm{H}]^{+}, 665\left[\mathrm{M}-\mathrm{CH}_{3} \mathrm{O}\right]^{+}, 559$ $\left[\mathrm{M}-\mathrm{CH}_{3} \mathrm{O}-\mathrm{PhCHO}\right]^{+}$

4,4'-((6R,7R,8R,8aS)-6-Methoxy-2-phenyl-hexahydropyrano[3,2-d][1,3]dioxine-7,8-diyl)bis(oxy)bis(methylene) bis(1-(4-nitrobenzyl)-1 H-1,2,3-triazole) (3g): White solid. mp 186 - $188{ }^{\circ} \mathrm{C}$. Yield: $91 \%$. ${ }^{1} \mathrm{H}$ NMR $\left(300 \mathrm{MHz}, \mathrm{CDCl}_{3}, J\right.$ in $\mathrm{Hz}) \delta 8.15\left(\mathrm{q}, 4 \mathrm{H},{ }^{3} J=8.5, \mathrm{Ar} \mathrm{H}\right), 7.89(\mathrm{~s}, 1 \mathrm{H}$, triazole $\mathrm{H}), 7.68$ (s, $1 \mathrm{H}$, triazole $\mathrm{H}), 7.43\left(\mathrm{~d}, 4 \mathrm{H},{ }^{3} J=8.2, \mathrm{Ar} \mathrm{H}\right), 7.34\left(\mathrm{t}, 4 \mathrm{H},{ }^{3} J=\right.$ 5.8, Ar H), $7.25\left(\mathrm{~d}, 2 \mathrm{H},{ }^{3} J=5.8, \mathrm{Ar} \mathrm{H}\right), 5.62\left(\mathrm{~s}, 2 \mathrm{H}, \mathrm{OCH}_{2}\right)$, 5.51 (s, 1H, Ph-CH), 5.44 (s, 2H, $\left.\mathrm{OCH}_{2}\right), 4.93-4.83$ (m, 5H, $\left.2 \mathrm{NCH}_{2}, 1-\mathrm{H}\right), 4.26\left(\mathrm{dd}, 1 \mathrm{H},{ }^{3} J_{6 \mathrm{eq}, 5}=3.8,{ }^{2} J_{6 \mathrm{eq}, 6 \mathrm{ax}}=9.3,6-\mathrm{H}_{\mathrm{eq}}\right)$, $3.90\left(\mathrm{t}, 1 \mathrm{H},{ }^{3} J_{3,4}={ }^{3} J_{3,2}=9.1,3-\mathrm{H}\right), 3.80\left(\mathrm{ddd}, 1 \mathrm{H},{ }^{3} J_{5,6 \mathrm{eq}}=3.8\right.$, $\left.{ }^{3} J_{5,4}=9.6,{ }^{3} J_{5,6 \mathrm{ax}}=10,5-\mathrm{H}\right), 3.70\left(\mathrm{t}, 1 \mathrm{H},{ }^{3} J_{6 \mathrm{ax}, 5}=10,{ }^{3} J_{6 \mathrm{ax}, 6 \mathrm{eq}}=\right.$ 9.7, 6- $\mathrm{H}_{\mathrm{ax}}$ ), 3.58-3.52 (m, 2H, 2-H, 4-H), 3.38 (s, 3H, OCH $\mathrm{OC}_{3}$; ${ }^{13} \mathrm{C} \mathrm{NMR}\left(75 \mathrm{MHz}, \mathrm{CDCl}_{3}\right) \delta 147.54,145.90,145.19,141.38$, $136.98,128.77,128.51,128.20,127.96,125.81,123.86,123.80$, $123.38,123.04,101.15,98.04,81.35,79.14,77.71,68.64,65.95$, 64.44, 61.90, 55.94, 52.71, 52.50; IR (KBr) v 3136, 3081, 2925, 2915, 2876, 1608, 1563, 1520, 1460, 1348, 1225, 1182, $1133,1109,1090,1051,1002 \mathrm{~cm}^{-1}$; MS $(\mathrm{m} / \mathrm{z}): 737[\mathrm{M}+\mathrm{Na}]^{+}$, $683\left[\mathrm{M}-\mathrm{CH}_{3} \mathrm{OH}\right]^{+}, 607\left[\mathrm{M}-\mathrm{PhCH}_{2} \mathrm{O}\right]^{+}$.

4,4'-((6R,7R,8R,8aS)-6-Methoxy-2-phenyl-hexahydropyrano[3,2-d][1,3]dioxine-7,8-diyl)bis(oxy)bis(methylene) bis(1-(4-fluorobenzyl)-1H-1,2,3-triazole) (3h): Syrup. Yield: $82 \%$. ${ }^{1} \mathrm{H}$ NMR $\left(300 \mathrm{MHz} \mathrm{CDCl}_{3}, J\right.$ in $\left.\mathrm{Hz}\right) \delta 7.72(\mathrm{~s}, 1 \mathrm{H}$, triazole $\mathrm{H}), 7.59(\mathrm{~s}, 1 \mathrm{H}$, triazole $\mathrm{H}), 7.46(\mathrm{~s}, 2 \mathrm{H}, \mathrm{Ar} \mathrm{H}), 7.36(\mathrm{~s}, 4 \mathrm{H}$, Ar H), 7.13 (s, 2H, Ar H), 7.14-7.11 (m, 3H, Ar H), 7.01-6.98 $(\mathrm{m}, 2 \mathrm{H}, \mathrm{Ar} \mathrm{H}), 5.56\left(\mathrm{~s}, 2 \mathrm{H}, \mathrm{OCH}_{2}\right), 5.51(\mathrm{~s}, 1 \mathrm{H}, \mathrm{Ph}-\mathrm{CH}), 5.43-$ $5.32\left(\mathrm{~m}, 2 \mathrm{H}, \mathrm{OCH}_{2}\right), 5.00-4.77\left(\mathrm{~m}, 5 \mathrm{H}, 2 \mathrm{NCH}_{2}, 1-\mathrm{H}\right), 4.26(\mathrm{dd}$, $\left.1 \mathrm{H},{ }^{3} \mathrm{~J}=4.3,{ }^{2} J_{6 \mathrm{eq}, 6 \mathrm{ax}}=9.3,6-\mathrm{H}_{\mathrm{eq}}\right), 3.92(\mathrm{~s}, 1 \mathrm{H}, 3-\mathrm{H}), 3.74-3.67$ (m, 2H, 5-H, 6- $\left.\mathrm{H}_{\mathrm{ax}}\right), 3.62-3.56(\mathrm{~m}, 2 \mathrm{H}, 2-\mathrm{H}, 4-\mathrm{H}), 3.35(\mathrm{~s}, 3 \mathrm{H}$, $\left.\mathrm{OCH}_{3}\right) ;{ }^{13} \mathrm{C} \mathrm{NMR}\left(75 \mathrm{MHz}, \mathrm{CDCl}_{3}\right) \delta 162.18,146.16,145.95$, $145.19,137.32,130.85,130.63,130.31,129.91,129.81,129.03$, $128.26,126.11,124.78,123.38,122.93,122.66,122.00,116.02$, $115.74,101.45,98.65,81.80,79.48,78.30,69.01,66.61,64.93$, 62.22, 55.18, 53.11, 47.63; MS $(\mathrm{m} / \mathrm{z}): 699[\mathrm{M}+\mathrm{K}]^{+}, 553$ [M$\left.\mathrm{PhCH}_{2} \mathrm{O}\right]^{+}$

4,4'-((6R,7R,8R,8aS)-6-Methoxy-2-phenyl-hexahydropyrano[3,2-d][1,3]dioxine-7,8-diyl)bis(oxy)bis(methylene) bis(1-(4-bromobenzyl)-1H-1,2,3-triazole) (3i): White solid. mp $168-170{ }^{\circ} \mathrm{C}$. Yield: $78 \%$. ${ }^{1} \mathrm{H}$ NMR $\left(300 \mathrm{MHz}, \mathrm{CDCl}_{3}, J\right.$ in $\mathrm{Hz}) \delta 7.70(\mathrm{~s}, 1 \mathrm{H}$, triazole $\mathrm{H}), 7.49(\mathrm{~s}, 1 \mathrm{H}$, triazole $\mathrm{H}), 7.42-7.35$ (m, 6H, Ar H), 7.30-7.29 (m, 3H, Ar H), 7.19 (s, 1H, Ar H), 
$7.10\left(\mathrm{~d}, 2 \mathrm{H},{ }^{3} \mathrm{~J}=8.3, \mathrm{Ar} \mathrm{H}\right), 6.95\left(\mathrm{~d}, 2 \mathrm{H},{ }^{3} J=8.3, \mathrm{Ar} \mathrm{H}\right), 5.45$ (s, $1 \mathrm{H}, \mathrm{Ph}-\mathrm{CH}), 5.38$ (d, $\left.2 \mathrm{H}, J=4.9, \mathrm{OCH}_{2}\right), 5.23\left(\mathrm{~s}, 2 \mathrm{H}, \mathrm{OCH}_{2}\right)$, 4.89-7.49 (m, 2H, $\mathrm{NCH}_{2}$ ), 4.74 (s, 3H, $\left.\mathrm{NCH}_{2}, 1-\mathrm{H}\right), 4.20$ (dd, $\left.1 \mathrm{H},{ }^{3} J_{6 \mathrm{eq}, 5}=4.2,{ }^{2} J_{6 \mathrm{eq}, 6 \mathrm{ax}}=9.4,6-\mathrm{H}_{\mathrm{eq}}\right), 3.84\left(\mathrm{t}, 1 \mathrm{H},{ }^{3} J_{4,3}={ }^{3} J_{2,3}=\right.$ $9.2,3-\mathrm{H}), 3.72\left(\mathrm{ddd}, 1 \mathrm{H},{ }^{3} J_{5,6 \mathrm{eq}}=4.1,{ }^{3} J_{5,4}=9.9,{ }^{3} J_{5,6 \mathrm{ax}}=10,5-\mathrm{H}\right)$, $3.64\left(\mathrm{t}, 1 \mathrm{H},{ }^{3} \mathrm{~J}_{6 \mathrm{ax}, 5}=10,6-\mathrm{H}_{\mathrm{ax}}\right), 3.54-3.45(\mathrm{~m}, 2 \mathrm{H}, 2-\mathrm{H}, 4-\mathrm{H}), 3.31$ $\left(\mathrm{s}, 3 \mathrm{H}, \mathrm{OCH}_{3}\right) ;{ }^{13} \mathrm{C} \mathrm{NMR}\left(75 \mathrm{MHz}, \mathrm{CDCl}_{3}\right) \delta 146.13,145.43$, $137.43,133.86,132.33,132.26,129.97,129.23,128.43,126.25$, $123.53,123.02,122.83,101.60,98.59,81.86,79.43,78.25$, 69.14, 66.54, 64.97, 62.35, 55.38, 53.51, 53.32; MS (m/z): 805 $[\mathrm{M}+\mathrm{Na}]^{+}, 749\left[\mathrm{M}-\mathrm{CH}_{3} \mathrm{OH}\right]^{+}$

4,4'-((6S,7S,8S,8aR)-6-Methoxy-2-phenyl-hexahydropyrano $[3,2-d][1,3]$ dioxine-7,8-diyl)bis(oxy)bis(methylene)bis(1octyl-1H-1,2,3-triazole) (6a): White solid. $\mathrm{mp} 78-81{ }^{\circ} \mathrm{C}$. Yield: $64 \%$. ${ }^{1} \mathrm{H} \mathrm{NMR}\left(300 \mathrm{MHz}, \mathrm{CDCl}_{3}, J\right.$ in $\left.\mathrm{Hz}\right) \delta 7.77(\mathrm{~s}, 1 \mathrm{H}$, triazole $\mathrm{H}), 7.58(\mathrm{~s}, 1 \mathrm{H}$, triazole $\mathrm{H}), 7.48\left(\mathrm{t}, 2 \mathrm{H},{ }^{3} J=5.2, \mathrm{Ph} 3-\mathrm{H}, 5-\mathrm{H}\right)$, 7.39 (t, 3H, ${ }^{3} J=5.2$, Ph 2-H, 4-H, 6-H), 5.53 (s, 1H, Ph-CH), $5.02-4.92\left(\mathrm{~m}, 2 \mathrm{H}, \mathrm{OCH}_{2}\right), 4.87\left(\mathrm{~s}, 2 \mathrm{H}, \mathrm{OCH}_{2}\right), 4.82(\mathrm{~d}, 1 \mathrm{H}$, $\left.{ }^{3} J_{1,2}=3.6,1-\mathrm{H}\right), 4.39-4.25\left(\mathrm{~m}, 3 \mathrm{H}, \mathrm{NCH}_{2}, 6-\mathrm{H}_{\mathrm{eq}}\right), 4.17(\mathrm{t}, 2 \mathrm{H}$, $\left.{ }^{3} J=7.3, \mathrm{NCH}_{2}\right), 3.96\left(\mathrm{t}, 1 \mathrm{H},{ }^{3} J_{4,3}={ }^{3} J_{3,2}=9.2,3-\mathrm{H}\right), 3.82(\mathrm{ddd}$, $\left.1 \mathrm{H},{ }^{3} J_{5,6 \mathrm{eq}}=4.8,{ }^{3} J_{5,4}=9.4,{ }^{3} J_{5,6 \mathrm{ax}}=10,5-\mathrm{H}\right), 3.72\left(\mathrm{t}, 1 \mathrm{H},{ }^{3} J_{6 \mathrm{ax}, 5}=\right.$ $\left.10,6-\mathrm{H}_{\mathrm{ax}}\right), 3.63\left(\mathrm{dd}, 1 \mathrm{H},{ }^{3} J_{2,1}=3.6,{ }^{3} J_{2,3}=9.2,2-\mathrm{H}\right), 3.57(\mathrm{t}, 1 \mathrm{H}$, $\left.{ }^{3} J_{4,3}=9.2,{ }^{3} J_{4,5}=9.4,4-\mathrm{H}\right), 3.40\left(\mathrm{~s}, 3 \mathrm{H}, \mathrm{OCH}_{3}\right), 1.90-1.86(\mathrm{~m}$, $\left.2 \mathrm{H}, \mathrm{CH}_{2}\right), 1.77-1.73\left(\mathrm{~m}, 2 \mathrm{H}, \mathrm{CH}_{2}\right), 1.29-1.24\left(\mathrm{~m}, 20 \mathrm{H}, 10 \mathrm{CH}_{2}\right)$, $0.86\left(\mathrm{t}, 6 \mathrm{H},{ }^{3} \mathrm{~J}=6.8,2 \mathrm{CH}_{3}\right) ;{ }^{13} \mathrm{C} \mathrm{NMR}\left(75 \mathrm{MHz}, \mathrm{CDCl}_{3}\right) \delta$ $145.38,144.58,137.30,128.99,128.20,126.08,123.10,122.51$, $101.45,98.56,81.63,79.26,78.17,68.98,66.59,64.86,62.22$, $55.20,50.29,50.13,31.62,30.21,30.16,28.96,28.87,26.43$, 26.39, 22.50, 13.97; IR (KBr) v 3128, 3150, 3091, 2954, 2922, 2851, 1547, 1465, 1445, 1376, 1326, 1281, 1177, 1154, 1086, $1045 \mathrm{~cm}^{-1}$; MS $(\mathrm{m} / \mathrm{z}): 691[\mathrm{M}+\mathrm{Na}]^{+}$.

4,4'-((6R,7S,8S,8aR)-6-Methoxy-2-phenyl-hexahydropyrano $[3,2-d][1,3]$ dioxine-7,8-diyl)bis(oxy)bis(methylene)bis(1-dodecyl-1H-1,2,3-triazole) (6b): White solid. mp $94-96^{\circ} \mathrm{C}$. Yield: $83 \%$. ${ }^{1} \mathrm{H} \mathrm{NMR}\left(300 \mathrm{MHz}, \mathrm{CDCl}_{3}, J\right.$ in $\left.\mathrm{Hz}\right) \delta 7.77(\mathrm{~s}, 1 \mathrm{H}$, triazole $\mathrm{H}), 7.58(\mathrm{~s}, 1 \mathrm{H}$, triazole $\mathrm{H}), 7.49\left(\mathrm{~d}, 2 \mathrm{H},{ }^{4} J=4.5, \mathrm{Ph} 3-\mathrm{H}, 5-\mathrm{H}\right)$, $7.38\left(\mathrm{~d}, 3 \mathrm{H},{ }^{4} J=4.5, \mathrm{Ph} 2-\mathrm{H}, 4-\mathrm{H}, 6-\mathrm{H}\right), 5.54$ (s, 1H, Ph CH), $4.98\left(\mathrm{~d}, 2 \mathrm{H},{ }^{4} \mathrm{~J}=4.2, \mathrm{OCH}_{2}\right), 4.87\left(\mathrm{~s}, 2 \mathrm{H}, \mathrm{OCH}_{2}\right), 4.82(\mathrm{~d}, 1 \mathrm{H}$, $\left.{ }^{3} J_{1,2}=3.3,1-\mathrm{H}\right), 4.35-4.26\left(\mathrm{~m}, 3 \mathrm{H}, \mathrm{NCH}_{2}, 6-\mathrm{H}_{\mathrm{eq}}\right), 4.18(\mathrm{t}, 2 \mathrm{H}$, $\left.{ }^{3} J=7.2, \mathrm{NCH}_{2} \mathrm{CH}_{2}\right), 3.97\left(\mathrm{t}, 1 \mathrm{H},{ }^{3} J_{4,3}={ }^{3} J_{3,2}=9.2,3-\mathrm{H}\right), 3.85$ (ddd, $\left.1 \mathrm{H},{ }^{3} J_{5,6 \mathrm{eq}}=4.8,{ }^{3} J_{5,4}=9.4,{ }^{3} J_{5,6 \mathrm{ax}}=10,5-\mathrm{H}\right), 3.72(\mathrm{t}, 1 \mathrm{H}$, $\left.{ }^{3} J_{6 \mathrm{ax}, 5}=10.0,6-\mathrm{H}_{\mathrm{ax}}\right), 3.63\left(\mathrm{dd}, 1 \mathrm{H},{ }^{3} J_{2,1}=3.6,{ }^{3} J_{2,3}=9.2,2-\mathrm{H}\right)$, $3.57\left(\mathrm{t}, 1 \mathrm{H},{ }^{3} J_{4,3}=9.2,{ }^{3} J_{4,5}=9.4,4-\mathrm{H}\right), 3.40\left(\mathrm{~s}, 3 \mathrm{H}, \mathrm{OCH}_{3}\right), 1.88$ (d, $\left.2 \mathrm{H}, \mathrm{NCH}_{2} \mathrm{CH}_{2}\right), 1.75$ (s, $4 \mathrm{H}, \mathrm{NCH}_{2} \mathrm{CH}_{2}$ including water), $1.30-1.25\left(\mathrm{~m}, 36 \mathrm{H}, 18 \mathrm{CH}_{2}\right), 0.88\left(\mathrm{t}, 6 \mathrm{H},{ }^{3} \mathrm{~J}=5.8,2 \mathrm{CH}_{3}\right) ;{ }^{13} \mathrm{C}$ NMR $\left(75 \mathrm{MHz} \mathrm{CDCl}_{3}\right) \delta 145.45,144.65,137.36,129.06$, $128.28,126.15,123.14,122.56,101.53,98.63,81.70,79.33$, $78.25,69.06,66.67,64.95,62.29,55.27,50.37,50.21,31.88$, $30.25,29.59,29.52,29.40,29.32,29.00,26.48,22.66,14.10$; IR $(\mathrm{KBr}) \vee 3135,3012,2954,2919,2849,1466,1376,1214$, $1148,1090,1056 \mathrm{~cm}^{-1}$; MS $(\mathrm{m} / \mathrm{z}): 819[\mathrm{M}+\mathrm{K}]^{+}, 803[\mathrm{M}+\mathrm{Na}]^{+}$, $747\left[\mathrm{M}-\mathrm{CH}_{3} \mathrm{O}\right]^{+}, 683\left[\mathrm{M}-\mathrm{PhCHO}-\mathrm{CH}_{3} \mathrm{O}\right]^{+}$.

General procedure for the deprotection of compounds 4a-i and 7a-b. A mixture of analytic grade concentrated hydrochloric acid $(1 \mathrm{~mL})$, distilled water $(5 \mathrm{~mL})$ and protected glucose derived 1,2,3-triazole $(110 \mathrm{mg})$ with a little methanol was stirred at room temperature for $24 \mathrm{~h}$. The solvents were evaporated under reduced pressure. The residue was washed with petroleum ether
(30 - $60{ }^{\circ} \mathrm{C}$ ) to remove benzaldehyde and dried to afford compounds $\mathbf{4 a - i}$ and $\mathbf{7 a - b}$.

$(3 S, 4 R, 5 R, 6 R)-4,5-B i s((1-(2-c h l o r o b e n z y l)-1 H-1,2,3-t r i-$ azol-4-yl)methoxy)-2-(hydroxymethyl)-6-methoxy-tetrahydro2H-pyran-3-ol hydrochloride (4a): Syrup. Yield: 99\%. ${ }^{1} \mathrm{H}$ NMR $\left(300 \mathrm{MHz} \mathrm{CDCl}_{3}, J\right.$ in $\left.\mathrm{Hz}\right) \delta 8.15(\mathrm{~s}, 1 \mathrm{H}$, triazole $\mathrm{H}), 8.12(\mathrm{~s}$, $1 \mathrm{H}$, triazole $\mathrm{H}), 7.48(\mathrm{~s}, 4 \mathrm{H}, \mathrm{Ar} \mathrm{H}), 7.30\left(\mathrm{~d}, 2 \mathrm{H},{ }^{3} J=7.9\right.$, Ar H), $7.21\left(\mathrm{~d}, 2 \mathrm{H},{ }^{3} \mathrm{~J}=8.0, \mathrm{Ar} \mathrm{H}\right), 5.66\left(\mathrm{~s}, 4 \mathrm{H}, 2 \mathrm{OCH}_{2}\right), 4.88(\mathrm{~s}, 2 \mathrm{H}$, $\mathrm{NCH}_{2}$ ), 4.72 (s, 3H, $\left.\mathrm{NCH}_{2}, 1-\mathrm{H}\right), 3.66-3.61$ (m, 3H, 5-H, 6-H), 3.40 (m, 3H, 2-H, 3-H, 4-H), 3.30 (s, 3H, OCH $) ;{ }^{13} \mathrm{C} \mathrm{NMR}(75$ $\left.\mathrm{MHz}, \mathrm{CDCl}_{3}\right) \delta 145.11,144.46,134.32,133.74,131.79,131.55$, 131.37, 129.01, 128.77, 127.64, 124.43, 97.19, 81.23, 79.07, $72.13,69.93,65.51,63.42,60.54,54.39,50.29,50.25$; IR (KBr) $v$ 3360, 2924, 2855, 2598, 2362, 1460, 1408, 1320, 1295, $1226,1168,1069,1004 \mathrm{~cm}^{-1}$; MS $(\mathrm{m} / \mathrm{z}): 627[\mathrm{M}-2 \mathrm{HCl}+\mathrm{Na}]^{+}$, $605[\mathrm{M}-2 \mathrm{HCl}+\mathrm{H}]^{+}$.

(3S,4R,5R,6R)-4,5-Bis((1-(2,4-dichlorobenzyl)-1H-1,2,3triazol-4-yl)methoxy)-2-(hydroxymethyl)-6-methoxy-tetrahydro-2H-pyran-3-ol hydrochloride (4b): Syrup. Yield: 98\%. ${ }^{1} \mathrm{H} \mathrm{NMR}\left(300 \mathrm{MHz}, \mathrm{CDCl}_{3}, J\right.$ in $\left.\mathrm{Hz}\right) \delta 8.15(\mathrm{~s}, 1 \mathrm{H}$, triazole $\mathrm{H})$, $8.12(\mathrm{~s}, 1 \mathrm{H}$, triazole $\mathrm{H}), 7.48(\mathrm{~s}, 2 \mathrm{H}, \mathrm{Ar} \mathrm{H}), 7.30\left(\mathrm{~d}, 2 \mathrm{H},{ }^{3} \mathrm{~J}=\right.$ 7.9, $\mathrm{Ar} \mathrm{H}), 7.20\left(\mathrm{~d}, 2 \mathrm{H},{ }^{3} J=7.9, \mathrm{Ar} \mathrm{H}\right), 5.66\left(\mathrm{~s}, 4 \mathrm{H}, \mathrm{OCH}_{2}\right)$, 4.88 (s, 2H, $\left.\mathrm{NCH}_{2}\right), 4.72$ (s, 3H, 3/2 $\left.\mathrm{NCH}_{2}, 1-\mathrm{H}\right), 4.23$ (bs, $1 \mathrm{H}$, $\mathrm{OH}), 3.66-3.63$ (m, 3H, 5-H, 6-H), 3.46 (s, 3H, 2-H, 3-H, 4-H), 3.30 (s, 3H, $\left.\mathrm{OCH}_{3}\right)$; IR ( $\left.\mathrm{KBr}\right) \vee 3391,2925,2361,1648,1591$, 1564, 1476, 1386, 1336, 1222, 1192, 1080, $1049 \mathrm{~cm}^{-1}$; MS $(\mathrm{m} / \mathrm{z}): 697[\mathrm{M}+\mathrm{Na}-2 \mathrm{HCl}]^{+}, 674[\mathrm{M}-2 \mathrm{HCl}]^{+}, 647[\mathrm{M}-2 \mathrm{HCl}-$ $\left.\mathrm{CH}_{3} \mathrm{OH}+\mathrm{H}\right]^{+}$.

$(3 S, 4 R, 5 R, 6 R)-4,5-B i s((1-(3,4-d i c h l o r o b e n z y l)-1 H-1,2,3-$ triazol-4-yl)methoxy)-2-(hydroxymethyl)-6-methoxy-tetrahydro-2H-pyran-3-ol hydrochloride (4c): Syrup. Yield: 100\%. ${ }^{1} \mathrm{H}$ NMR $\left(300 \mathrm{MHz}, \mathrm{d}^{6}-\mathrm{DMSO}, J\right.$ in $\left.\mathrm{Hz}\right) \delta 8.21$ (s, $1 \mathrm{H}$, triazole H), 7.53-7.45 (m, 4H, Ar H), 7.27 (d, 2H, $\left.{ }^{3} J=7.7, \operatorname{Ar~H}\right), 5.58$ $\left(\mathrm{s}, 4 \mathrm{H}, 2 \mathrm{OCH}_{2}\right), 4.88\left(\mathrm{~s}, 5 \mathrm{H}, 2 \mathrm{NCH}_{2}, 1-\mathrm{H}\right), 3.70-3.67(\mathrm{~m}, 3 \mathrm{H}$, 5-H, 6-H), 3.47-3.37 (m, 3H, 2-H, 3-H, 4-H), 3.32 (s, 3H, $\left.\mathrm{OCH}_{3}\right) ; \mathrm{MS}(\mathrm{m} / \mathrm{z}): 771[\mathrm{M}+\mathrm{Na}]^{+}, 697[\mathrm{M}+\mathrm{Na}-2 \mathrm{HCl}]^{+}$.

(3S,4R,5R,6R)-4,5-Bis((1-(3-chlorobenzyl)-1H-1,2,3-triazol-4-yl)methoxy)-2-(hydroxymethyl)-6-methoxy-tetrahydro2H-pyran-3-ol hydrochloride (4d): Syrup. Yield: 97\%. ${ }^{1} \mathrm{H}$ NMR $\left(300 \mathrm{MHz} \mathrm{CDCl}_{3}, J\right.$ in $\left.\mathrm{Hz}\right) \delta 8.21(\mathrm{~s}, 1 \mathrm{H}$, triazole $\mathrm{H}), 8.04(\mathrm{~s}$, $1 \mathrm{H}$, triazole $\mathrm{H}), 7.48(\mathrm{~s}, 1 \mathrm{H}, \mathrm{Ar} \mathrm{H}), 7.35-7.24(\mathrm{~m}, 7 \mathrm{H}, \mathrm{Ar} \mathrm{H})$, $5.57\left(\mathrm{~d}, 4 \mathrm{H},{ }^{3} \mathrm{~J}=11.6,2 \mathrm{OCH}_{2}\right), 5.03-4.96\left(\mathrm{~m}, 2 \mathrm{H}, \mathrm{NCH}_{2}\right), 4.80$ (s, 3H, $\left.\mathrm{NCH}_{2}, 1-\mathrm{H}\right), 3.84-3.76$ (m, 3H, 5-H, 6-H), 3.73-3.51 (m, 3H, 2-H, 3-H, 4-H), $3.34\left(\mathrm{~s}, 3 \mathrm{H}, \mathrm{OCH}_{3}\right)$; MS $(\mathrm{m} / \mathrm{z}): 627$ $[\mathrm{M}-2 \mathrm{HCl}+\mathrm{Na}]^{+}, 605[\mathrm{M}-2 \mathrm{HCl}+\mathrm{H}]^{+}$

(3S,4R,5R,6R)-4,5-Bis((1-(4-chlorobenzyl)-1H-1,2,3-triazol-4-yl)methoxy)-2-(hydroxymethyl)-6-methoxy-tetrahydro2H-pyran-3-ol hydrochloride (4e): Syrup. Yield: 98\%. ${ }^{1} \mathrm{H}$ NMR (300 MHz, d $\mathrm{d}^{6}$-DMSO, $J$ in Hz) $\delta 8.19$ (s, $1 \mathrm{H}$, triazole $\left.\mathrm{H}\right), 8.15$ $(\mathrm{s}, 1 \mathrm{H}$, triazole $\mathrm{H}), 7.41\left(\mathrm{~d}, 4 \mathrm{H},{ }^{3} \mathrm{~J}=8.1, \mathrm{Ar} \mathrm{H}\right), 7.31\left(\mathrm{~d}, 4 \mathrm{H},{ }^{3} \mathrm{~J}=\right.$ 8.1, $\mathrm{ArH}$ ), 5.57 (d, $\left.4 \mathrm{H},{ }^{4} \mathrm{~J}=2.7,2 \mathrm{OCH}_{2}\right), 4.79\left(\mathrm{~s}, 2 \mathrm{H}, \mathrm{NCH}_{2}\right)$, 4.68-4.65 (m, 3H, $\left.\mathrm{NCH}_{2}, 1-\mathrm{H}\right), 3.60\left(\mathrm{~d}, 1 \mathrm{H},{ }^{3} \mathrm{~J}=11.4,3-\mathrm{H}\right)$, 3.54-3.43 (m, 2H, 6-H), 3.35-3.26 (m, 2H, 2-H, 4-H), 3.22 (s, $\left.3 \mathrm{H}, \mathrm{OCH}_{3}\right) ;{ }^{13} \mathrm{C}$ NMR (75 MHz, d $\mathrm{d}^{6}$-DMSO) $\delta 145.77,145.43$, 135.12, 132.85, 129.88, 128.77, 124.20, 123.98, 97.07, 81.35, 78.86, 72.58, 69.69, 65.70, 63.29, 60.56, 54.19, 52.00; IR (KBr) v 3426, 3121, 3105, 2917, 2848.6, 1607, 1548, 1510, 1255, $1181,1045 \mathrm{~cm}^{-1}$; MS $(\mathrm{m} / z): 697[\mathrm{M}-2 \mathrm{HCl}+\mathrm{Na}]^{+}$. 
$(3 S, 4 R, 5 R, 6 R)-4,5-B i s((1-(2,4-d i f l u o r o b e n z y l)-1 H-1,2,3-$ triazol-4-yl)methoxy)-2-(hydroxymethyl)-6-methoxy-tetrahydro-2H-pyran-3-ol hydrochloride (4f): Syrup. Yield: 98\%. $\mathrm{H} \mathrm{NMR}\left(300 \mathrm{MHz}, \mathrm{CDCl}_{3}, J\right.$ in $\left.\mathrm{Hz}\right) \delta 8.23(\mathrm{~s}, 2 \mathrm{H}$, triazole $\mathrm{H})$, 7.65-7.37 (m, 4H, Ar H), 6.90 (s, 2H, Ar H), 5.57 (s, 4H, $\mathrm{OCH}_{2}$ ), 4.93-4.76 (m, 5H, $\left.\mathrm{NCH}_{2}, 1-\mathrm{H}\right), 3.74(\mathrm{~s}, 3 \mathrm{H}, 3-\mathrm{H}, 6-\mathrm{H}) 3.51-3.42$ (m, 3H, 2-H, 4-H, 5-H), 3.37 (s, 3H, $\left.\mathrm{OCH}_{3}\right)$; IR (KBr) v 3385, 2926, 2361, 2103, 1620, 1509, 1434, 1335, 1277, 1223, 1193, $1104.9,1054 \mathrm{~cm}^{-1}$; MS $(\mathrm{m} / z): 631[\mathrm{M}-2 \mathrm{HCl}+\mathrm{Na}]^{+}$.

(3S,4R,5R,6R)-2-(Hydroxymethyl)-6-methoxy-4,5-bis((1(4-nitrobenzyl)-1H-1,2,3-triazol-4-yl)methoxy)-tetrahydro2H-pyran-3-ol hydrochloride (4g): Syrup. Yield: 94\%. ${ }^{1} \mathrm{H}$ NMR (300 MHz, $\mathrm{CDCl}_{3}, J$ in Hz) $\delta 8.26-8.18(\mathrm{~m}, 6 \mathrm{H}, \mathrm{Ar} \mathrm{H}), 7.48$ (s, $4 \mathrm{H}$, triazole $\mathrm{H}, \mathrm{Ar} \mathrm{H}), 5.76\left(\mathrm{~s}, 4 \mathrm{H}, 2 \mathrm{OCH}_{2}\right), 4.82\left(\mathrm{~s}, 2 \mathrm{H}, \mathrm{NCH}_{2}\right)$, 4.72-4.69 (m, 3H, 2NCH, $1-\mathrm{H}), 3.65-3.49$ (m, 4H, 3-H, 5- $\mathrm{H}$, 6-H), 3.37-3.27 (m, 2H, 2-H, 4-H), $3.24\left(\mathrm{~s}, 3 \mathrm{H}, \mathrm{OCH}_{3}\right) ;{ }^{13} \mathrm{C}$ NMR $\left(75 \mathrm{MHz}, \mathrm{CDCl}_{3}\right) \delta 147.19,145.53,144.86,143.46,128.94$, $124.51,123.85,97.04,82.27,81.24,78.85,72.55,69.74,65.62$, 64.88, 63.28, 60.57, 54.20, 51.88; IR (KBr) v 3406, 3142, 3078, 2925, 1608, 1527, 1522, 1461, 1348, 1225, 1187, 1110, $1050 \mathrm{~cm}^{-1}$; MS $(\mathrm{m} / \mathrm{z}): 649[\mathrm{M}+\mathrm{Na}]^{+}, 595\left[\mathrm{M}-\mathrm{CH}_{3} \mathrm{O}\right]^{+}$

(3S,4R,5R,6R)-4,5-Bis((1-(4-fluorobenzyl)-1H-1,2,3-triazol-4-yl)methoxy)-2-(hydroxymethyl)-6-methoxy-tetrahydro2H-pyran-3-ol hydrochloride (4h): Syrup. Yield: 97\%. ${ }^{1} \mathrm{H}$ NMR $\left(300 \mathrm{MHz} \mathrm{CDCl}_{3}, J\right.$ in $\left.\mathrm{Hz}\right) \delta 8.14(\mathrm{~s}, 1 \mathrm{H}$, triazole $\mathrm{H}), 8.11(\mathrm{~s}$, $1 \mathrm{H}$, triazole $\mathrm{H}), 7.39-7.18(\mathrm{~m}, 8 \mathrm{H}, \mathrm{Ar} \mathrm{H}), 5.64\left(\mathrm{~s}, 2 \mathrm{H}, \mathrm{OCH}_{2}\right)$, $5.49\left(\mathrm{~s}, 2 \mathrm{H}, \mathrm{OCH}_{2}\right), 4.78\left(\mathrm{~s}, 2 \mathrm{H}, \mathrm{NCH}_{2}\right), 4.65\left(\mathrm{~s}, 3 \mathrm{H}, \mathrm{NCH}_{2}\right.$, 1-H), 3.63-3.49 (m, 4H, 3-H, 5-H, 6-H), 3.36-3.24 (m, 2H, 2-H, 4-H), 3.21 (s, 3H, OCH $)$; MS (m/z): $595[\mathrm{M}+\mathrm{Na}-2 \mathrm{HCl}]^{+}$.

$(3 S, 4 R, 5 R, 6 R)-4,5-B i s((1-(4-b r o m o b e n z y l)-1 H-1,2,3-t r i-$ azol-4-yl)methoxy)-2-(hydroxymethyl)-6-methoxy-tetrahydro2H-pyran-3-ol hydrochloride (4i): Syrup. Yield: 94\%. ${ }^{1}$ H NMR $\left(300 \mathrm{MHz}, \mathrm{d}^{6}-\mathrm{DMSO}, J\right.$ in $\left.\mathrm{Hz}\right) \delta 8.22(\mathrm{~s}, 1 \mathrm{H}$, triazole $\mathrm{H}), 8.18$ $(\mathrm{s}, 1 \mathrm{H}$, triazole $\mathrm{H}), 7.55\left(\mathrm{~d}, 4 \mathrm{H},{ }^{3} \mathrm{~J}=7.8, \mathrm{Ar} \mathrm{H}\right), 7.26\left(\mathrm{~d}, 4 \mathrm{H},{ }^{3} \mathrm{~J}=\right.$ 7.8, $\mathrm{Ar} \mathrm{H}), 5.57$ (s, 6H, 2OCH, $2 \mathrm{OH}), 4.80$ (s, 3H, $\left.\mathrm{NCH}_{2}, 1-\mathrm{H}\right)$, $4.67\left(\mathrm{~d}, 3 \mathrm{H},{ }^{3} \mathrm{~J}=7.5, \mathrm{NCH}_{2}, 3-\mathrm{H}\right), 3.63-3.45(\mathrm{~m}, 3 \mathrm{H}, 5-\mathrm{H}, 6-\mathrm{H})$, 3.34-3.27 (m, 2H, 2-H, 4-H), $3.22\left(\mathrm{~s}, 3 \mathrm{H}, \mathrm{OCH}_{3}\right) ;{ }^{13} \mathrm{C} \mathrm{NMR}$ (75 MHz, d $\mathrm{d}^{6}$-DMSO) $\delta 145.42,144.79,135.57,131.78,130.29$, $124.36,124.19,121.52,97.12,81.38,78.91,72.64,69.71,65.73$, $63.34,60.58,54.28,52.17 ; \mathrm{MS}(\mathrm{m} / \mathrm{z}): 595[\mathrm{M}-2 \mathrm{HCl}+\mathrm{Na}]^{+}, 573$ $[\mathrm{M}-2 \mathrm{HCl}+\mathrm{H}]^{+}$.

$(3 R, 4 S, 5 S, 6 R)-2-(H y d r o x y m e t h y l)-6-m e t h o x y-4,5-b i s((1-$ octyl-1 $H$-1,2,3-triazol-4-yl)methoxy)-tetrahydro-2H-pyran3-ol hydrochloride (7a): Syrup. Yield: $98 \%$. ${ }^{1} \mathrm{H}$ NMR $(300$ $\mathrm{MHz}, \mathrm{d}^{6}$-DMSO, $J$ in $\left.\mathrm{Hz}\right) \delta 8.12(\mathrm{~s}, 1 \mathrm{H}$, triazole $\mathrm{H}), 8.07(\mathrm{~s}$, $1 \mathrm{H}$, triazole $\mathrm{H}), 4.79\left(\mathrm{~s}, 2 \mathrm{H}, \mathrm{OCH}_{2}\right), 4.65\left(\mathrm{~s}, 2 \mathrm{H}, \mathrm{OCH}_{2}\right), 4.29$ (s, 5H, 2NCH, $1-\mathrm{H}), 3.68-3.54(\mathrm{~m}, 3 \mathrm{H}, 5-\mathrm{H}, 6-\mathrm{H}), 3.50-3.43$ (m, $2 \mathrm{H}, 2-\mathrm{H}, 3-\mathrm{H}), 3.34-3.31$ (m, 1H, 4-H, ), 3.24 (s, 3H, OCH $)$, $1.77\left(\mathrm{~s}, 4 \mathrm{H}, 2 \mathrm{NCH}_{2} \mathrm{CH}_{2}\right), 1.21\left(\mathrm{~s}, 20 \mathrm{H}, 10 \mathrm{CH}_{2}\right), 0.81(\mathrm{~d}, 6 \mathrm{H}$, $\left.{ }^{3} \mathrm{~J}=6.2,2 \mathrm{CH}_{3}\right) ;{ }^{13} \mathrm{C} \mathrm{NMR}\left(75 \mathrm{MHz}, \mathrm{d}^{6}\right.$-DMSO) $\delta 144.97$, 144.29, 123.60, 97.11, 81.38, 78.79, 76.56, 69.81, 65.77, 63.34, $60.62,54.25,49.40,31.24,29.77,28.59,28.43,25.92,22.11$, 13.99; IR (KBr) v 3426, 3159, 3011, 2967, 2871, 1451, 1435, $1167,1090,1053 \mathrm{~cm}^{-1}$; MS $(\mathrm{m} / \mathrm{z}): 603[\mathrm{M}-2 \mathrm{HCl}+\mathrm{Na}]^{+}, 581$ $[\mathrm{M}-2 \mathrm{HCl}+\mathrm{H}]^{+}$.

4,5-Bis((1-dodecyl-1H-1,2,3-triazol-4-yl)methoxy)-2-(hydroxymethyl)-6-methoxy-tetrahydro-2H-pyran-3-ol hydrochloride (7b): White Solid. mp $63-65{ }^{\circ} \mathrm{C}$. Yield: 98\%. ${ }^{1} \mathrm{H}$ NMR $\left(300 \mathrm{MHz}, \mathrm{d}^{6}\right.$-DMSO, $J$ in $\left.\mathrm{Hz}\right) \delta 8.11(\mathrm{~s}, 1 \mathrm{H}$, triazole $\mathrm{H}), 8.06$ (s, $1 \mathrm{H}$, triazole $\mathrm{H}), 4.79\left(\mathrm{~d}, 2 \mathrm{H},{ }^{4} \mathrm{~J}=2.7, \mathrm{OCH}_{2}\right), 4.72(\mathrm{~d}, 1 \mathrm{H}$, $\left.{ }^{4} J=2.9,1-\mathrm{H}\right), 4.66\left(\mathrm{~s}, 2 \mathrm{H}, \mathrm{OCH}_{2}\right), 4.31\left(\mathrm{~d}, 4 \mathrm{H}, 2 \mathrm{NCH}_{2}\right)$, 3.66-3.61 (m, 2H, 6-H), 3.52-3.47 (m, 2H, 2-H, 3-H), 3.33 (d, $\left.2 \mathrm{H},{ }^{3} J_{4,3}=9.6,4-\mathrm{H}, 5-\mathrm{H}\right), 3.26\left(\mathrm{~s}, 3 \mathrm{H}, \mathrm{OCH}_{3}\right), 1.78(\mathrm{~s}, 4 \mathrm{H}$, $\left.2 \mathrm{NCH}_{2} \mathrm{CH}_{2}\right), 1.22\left(\mathrm{~s}, 36 \mathrm{H}, 18 \mathrm{CH}_{2}\right), 0.84\left(\mathrm{~d}, 6 \mathrm{H},{ }^{3} \mathrm{~J}=6.5,2 \mathrm{CH}_{3}\right)$; ${ }^{13} \mathrm{C}$ NMR (75 MHz, d ${ }^{6}$-DMSO) $\delta 145.05,144.38,123.92$, $123.69,97.12,81.41,78.85,72.60,69.85,65.84,63.38,60.62$, 54.35, 49.49, 31.46, 29.85, 29.17, 29.04, 28.87, 28.57, 26.00, 22.26, 14.09; IR (KBr) v 3415, 3145, 3100, 2950, 2916, 2849 $1465,1388,1163,1052,779,720 \mathrm{~cm}^{-1}$; MS $(\mathrm{m} / \mathrm{z}): 715$ [M+Na$2 \mathrm{HCl}]^{+}, 693[\mathrm{M}-2 \mathrm{HCl}+\mathrm{H}]^{+}$.

General procedure for the synthesis of target compounds 5a-i and 8a-b. The deprotected $\alpha$-D-glucoside bis-1,2,3-triazole hydrochloride $(100 \mathrm{mg})$ was dissolved in water $(5 \mathrm{~mL})$. The resulting solution was neutralized with $25 \%$ analytic grade ammonium hydroxide under stirring, and then the mixture was evaporated to dryness. The solid residue was washed with $\mathrm{CHCl}_{3}(20 \mathrm{~mL} \times 3)$. The combined $\mathrm{CHCl}_{3}$ solution was evaporated to afford the pure compounds $\mathbf{5 a - i}$ and $\mathbf{8 a - b}$.

$(3 S, 4 R, 5 R, 6 R)-4,5-B i s((1-(2-c h l o r o b e n z y l)-1 H-1,2,3-t r i-$ azol-4-yl)methoxy)-2-(hydroxymethyl)-6-methoxy-tetrahydro2H-pyran-3-ol (5a): Syrup. Yield: 99\%. ${ }^{1} \mathrm{H}$ NMR (300 MHz, $\mathrm{CDCl}_{3}, J$ in $\left.\mathrm{Hz}\right) \delta 7.66(\mathrm{~s}, 1 \mathrm{H}$, triazole $\mathrm{H}), 7.60(\mathrm{~s}, 1 \mathrm{H}$, triazole H), 7.40-7.38 (m, 2H, Ar H), $7.31-7.18$ (m, 6H, Ar H), 5.60 (s, $\left.4 \mathrm{H}, 2 \mathrm{OCH}_{2}\right), 4.97\left(\mathrm{~d}, 1 \mathrm{H},{ }^{3} \mathrm{~J}=12.7,1 / 2 \mathrm{NCH}_{2}\right), 4.84-4.74(\mathrm{~m}$, $\left.4 \mathrm{H}, 3 / 2 \mathrm{NCH}_{2}, 1-\mathrm{H}\right), 3.80-3.72(\mathrm{~m}, 3 \mathrm{H}, 5-\mathrm{H}, 6-\mathrm{H}), 3.64-3.59$ (m, 2H, 2-H, 3-H), 3.53-3.49 (m, 1H, 4-H), 3.32 (s, 3H, OCH ; $_{3}$ ${ }^{13} \mathrm{C} \mathrm{NMR}\left(75 \mathrm{MHz}_{2} \mathrm{CDCl}_{3}\right) \delta 145.81,145.21,133.62,132.48$, $132.32,130.58,130.42,130.02,127.74,123.40,122.75,97.92$, $82.33,79.80,71.18,70.88,65.75,64.52,62.61,55.13,51.64$, 51.54; IR (KBr) v 3360, 2926, 2361, 1649, 1559, 1472, 1402, $1337,1192,1110,1052 \mathrm{~cm}^{-1}$; MS $(\mathrm{m} / \mathrm{z}): 627[\mathrm{M}+\mathrm{Na}]^{+}, 605$ $[\mathrm{M}+\mathrm{H}]^{+}$

$(3 S, 4 R, 5 R, 6 R)-4,5-B i s((1-(2,4-d i c h l o r o b e n z y l)-1 H-1,2,3-$ triazol-4-yl)methoxy)-2-(hydroxymethyl)-6-methoxy-tetrahydro-2H-pyran-3-ol (5b): White solid. mp $72-73{ }^{\circ} \mathrm{C}$. Yield: $100 \% .{ }^{1} \mathrm{HNMR}\left(300 \mathrm{MHz}, \mathrm{CDCl}_{3}, J\right.$ in $\left.\mathrm{Hz}\right) \delta 7.76(\mathrm{~s}, 2 \mathrm{H}$, triazole H), 7.42-7.39 (m, 2H, Ar H), 7.29-7.12 (m, 4H, Ar H), 5.58 (d, $\left.4 \mathrm{H}, J=11.2,2 \mathrm{OCH}_{2}\right), 4.98-4.76\left(\mathrm{~m}, 5 \mathrm{H}, 2 \mathrm{NCH}_{2}, 1-\mathrm{H}\right), 4.00$ (s, 3-H, 2OH including water), 3.80-3.75 (m, 3H, 5-H, 6-H), 3.61-3.50 (m, 3H, 2-H, 3-H, 4-H), 3.35 (s, 3H, OCH $) ;{ }^{13} \mathrm{C} \mathrm{NMR}$ $\left(100 \mathrm{MHz}, \mathrm{CDCl}_{3}\right) \delta 145.50,144.97,135.52,134.11,131.23$, $130.92,130.74,129.65,127.87,123.51,123.20,97.67,81.90$, 79.50, 71.16, 70.34, 65.52, 64.15, 61.96, 54.97, 50.97, 50.80; IR $(\mathrm{KBr}) \vee 3417,3142,2923,2362,2101,1620,1591,1563$, $1475,1388,1224,1194 \mathrm{~cm}^{-1}$; MS (m/z): $697[\mathrm{M}+\mathrm{Na}]^{+}, 674[\mathrm{M}]^{+}$, $647\left[\mathrm{M}-\mathrm{CH}_{3} \mathrm{OH}+\mathrm{H}\right]^{+}$.

(3S,4R,5R,6R)-4,5-Bis((1-(3,4-dichlorobenzyl)-1H-1,2,3triazol-4-yl)methoxy)-2-(hydroxymethyl)-6-methoxy-tetrahydro-2H-pyran-3-ol (5c): White solid. mp $74-75^{\circ} \mathrm{C}$. Yield: $100 \% .{ }^{1} \mathrm{HNMR}\left(300 \mathrm{MHz}, \mathrm{CDCl}_{3}, J\right.$ in $\left.\mathrm{Hz}\right) \delta 7.73(\mathrm{~s}, 1 \mathrm{H}$, triazole $\mathrm{H}), 7.70(\mathrm{~s}, 1 \mathrm{H}$, triazole H), 7.43-7.37 (m, 4H, Ar H), 7.10 (d, $2 \mathrm{H},{ }^{3} \mathrm{~J}=7.9$, Ar H), 5.45 (s, 4H, 20CH $), 4.99-4.73(\mathrm{~m}, 5 \mathrm{H}$, $\left.2 \mathrm{NCH}_{2}, 1-\mathrm{H}\right), 3.80-3.73$ (m, 3H, 5-H, 6-H), 3.66-3.49 (m, 3H, 2-H, 3-H, 4-H), 3.36 (s, 3H, OCH $H_{3}$ ), 3.14 (bs, 4H, 2OH including water) ${ }^{13} \mathrm{C}$ NMR $\left(100 \mathrm{MHz}, \mathrm{CDCl}_{3}\right) \delta 145.86,145.28,134.67$, $134.53,133.17,133.09,131.06,130.00,129.95,127.35,123.29$, 
$122.92,97.60,81.85,79.49,71.10,70.40,70.30,65.69,64.13$, 61.98, 55.03, 52.86, 52.79; IR (KBr) v 3442, 2923, 2853, 2361, 2338, 1650, 1457, 1192, $1124 \mathrm{~cm}^{-1}$; MS $(\mathrm{m} / \mathrm{z}): 697[\mathrm{M}+\mathrm{Na}]^{+}$, $595\left[\mathrm{M}+\mathrm{Na}-\mathrm{CH}_{3} \mathrm{OH}\right]^{+}$.

(3S,4R,5R,6R)-4,5-Bis((1-(3-chlorobenzyl)-1H-1,2,3-triazol-4-yl)methoxy)-2-(hydroxymethyl)-6-methoxy-tetrahydro2H-pyran-3-ol (5d): Syrup. Yield: 96\%. ${ }^{1} \mathrm{H}$ NMR (300 MHz, $\mathrm{CDCl}_{3}, J$ in $\left.\mathrm{Hz}\right) \delta 7.58(\mathrm{~s}, 1 \mathrm{H}$, triazole $\mathrm{H}), 7.44(\mathrm{~s}, 1 \mathrm{H}$, triazole $\mathrm{H})$, 7.25-7.20 (m, 6H, Ar H), 7.08 (d, 2H, ${ }^{3} J=6.3$, Ar H), 5.41-5.35 $\left(\mathrm{m}, 4 \mathrm{H}, 2 \mathrm{OCH}_{2}\right), 4.80\left(\mathrm{~d}, 1 \mathrm{H},{ }^{3} \mathrm{~J}=12.8,1 / 2 \mathrm{NCH}_{2}\right), 4.76-4.71$ (m, $\left.4 \mathrm{H}, 3 / 2 \mathrm{NCH}_{2}, 1-\mathrm{H}\right), 3.76-3.67$ (m, 3H, 5-H, 6-H), 3.54-3.52 (m, 2H, 2-H, 3-H), 3.48-3.43 (m, 1H, 4-H), 3.28 (s, 3H, OCH $\mathrm{OH}_{3}$; ${ }^{13} \mathrm{C} \mathrm{NMR}\left(100 \mathrm{MHz}, \mathrm{CDCl}_{3}\right) \delta 146.22,145.56,136.46,136.68$, 135.20, 131.06, 130.59, 129.23, 129.15, 128.35, 126.36, 123.17, $122.31,97.89,82.37,79.83,76.74,71.08,65.85,64.53,62.90$, 55.20, 53.69, 53.59; IR (KBr) v 3383, 2360, 2338, 2101, 1648, 1455, 1195, $1106 \mathrm{~cm}^{-1}$; MS (m/z): $627[\mathrm{M}+\mathrm{Na}]^{+}, 605[\mathrm{M}+\mathrm{H}]^{+}$.

(3S,4R,5R,6R)-4,5-Bis((1-(4-chlorobenzyl)-1H-1,2,3-triazol-4-yl)methoxy)-2-(hydroxymethyl)-6-methoxy-tetrahydro2H-pyran-3-ol (5e): White solid. mp $66-68{ }^{\circ} \mathrm{C}$. Yield: $100 \%$. ${ }^{1} \mathrm{H}$ NMR $\left(300 \mathrm{MHz}, \mathrm{CDCl}_{3}, J\right.$ in $\left.\mathrm{Hz}\right) \delta 7.61(\mathrm{~s}, 2 \mathrm{H}$, triazole $\mathrm{H})$, 7.20-7.16 (m, 4H, Ar H), 7.10-7.07 (m, 4H, Ar H), 5.32 (d, 4H, $\left.{ }^{3} J=5.0,2 \mathrm{OCH}_{2}\right), 4.86-4.72\left(\mathrm{~m}, 2 \mathrm{H}, \mathrm{NCH}_{2}\right), 4.67\left(\mathrm{~d}, 1 \mathrm{H},{ }^{3} \mathrm{~J}=\right.$ 3.0, 1-H), 4.64 (s, 2H, NCH$), 4.16$ (bs, $2 \mathrm{H}, 2 \mathrm{OH}), 3.76-3.62$ (m, 3H, 5-H, 6-H), 3.57-3.45 (m, 2H, 2-H, 3-H), 3.39 (dd, 1H, $\left.{ }^{4} J=2.9,{ }^{3} J=9.1,4-\mathrm{H}\right), 3.22$ (s, $\left.3 \mathrm{H}, \mathrm{OCH}_{3}\right) ;{ }^{13} \mathrm{C} \mathrm{NMR}(75 \mathrm{MHz}$, $\left.\mathrm{CDCl}_{3}\right) \delta 145.55,144.96,134.35,132.91,129.28,128.96$, 126.00, 123.10, 122.81, 97.41, 82.59, 79.32, 76.57, 69.83, 69.92, 71.12, 65.58, 63.94, 61.45, 54.75, 53.05; IR (KBr) v 3428, 3100, 3010, 2919, 1600, 1493, 1460, 1411, 1336, 1223, 1193, 1128, 1090, $1017 \mathrm{~cm}^{-1}$; MS $(\mathrm{m} / \mathrm{z}): 627[\mathrm{M}+\mathrm{Na}]^{+}$

(3S,4R,5R,6R)-4,5-Bis((1-(2,4-difluorobenzyl)-1H-1,2,3triazol-4-yl)methoxy)-2-(hydroxymethyl)-6-methoxy-tetrahydro-2H-pyran-3-ol (5f): White solid. mp $79-80^{\circ} \mathrm{C}$. Yield: 97\%. ${ }^{1} \mathrm{H}$ NMR $\left(400 \mathrm{MHz}, \mathrm{CDCl}_{3}, J\right.$ in $\left.\mathrm{Hz}\right) \delta 8.01(\mathrm{~s}, 1 \mathrm{H}$, triazole $\mathrm{H}), 7.85(\mathrm{~s}, 1 \mathrm{H}$, triazole $\mathrm{H}), 7.37-7.33(\mathrm{~m}, 4 \mathrm{H}, \mathrm{Ar} \mathrm{H}), 7.10(\mathrm{t}$, $\left.2 \mathrm{H},{ }^{3} J=8.4, \mathrm{Ar} \mathrm{H}\right), 5.44$ (d, $\left.4 \mathrm{H}, J=7.2,2 \mathrm{OCH}_{2}\right), 5.10$ (bs, $4 \mathrm{H}$, $4 \mathrm{OH}), 4.93-4.83\left(\mathrm{~m}, 2 \mathrm{H}, \mathrm{NCH}_{2}\right), 4.74-4.66\left(\mathrm{~m}, 3 \mathrm{H}, \mathrm{NCH}_{2}, 1-\mathrm{H}\right)$, $3.80\left(\mathrm{~d}, 1 \mathrm{H},{ }^{3} \mathrm{~J}=8.4,5-\mathrm{H}\right), 3.71-3.66(\mathrm{~m}, 2 \mathrm{H}, 6-\mathrm{H}), 3.60(\mathrm{t}$, $\left.1 \mathrm{H},{ }^{3} \mathrm{~J}=9.2,3-\mathrm{H}\right), 3.49-3.42$ (m, 2H, 2-H, 4-H), 3.27 (s, 3H, $\left.\mathrm{OCH}_{3}\right) ;{ }^{13} \mathrm{C} \mathrm{NMR}\left(100 \mathrm{MHz}, \mathrm{CDCl}_{3}\right) \delta 145.02,144.80,134.52$, 134.21, 132.97, 132.95, 132.91, 132.89, 130.95, 130.93, 130.10, 129.96, 127.59, 127.43, 124.12, 123.80, 97.40, 81.61, 79.34, $71.31,69.79,65.18,63.67,61.26,54.90,53.13,52.82$; IR (KBr) $v 3441,2925,2854,2306,1649,1456,1338,1222,1192,1110$, $1049 \mathrm{~cm}^{-1} ; \mathrm{MS}(\mathrm{m} / \mathrm{z}): 647[\mathrm{M}+\mathrm{K}]^{+}, 631[\mathrm{M}+\mathrm{Na}]^{+}$.

(3S,4R,5R,6R)-2-(Hydroxymethyl)-6-methoxy-4,5-bis((1(4-nitrobenzyl)-1H-1,2,3-triazol-4-yl)methoxy)-tetrahydro2H-pyran-3-ol (5g): Syrup. Yield: 98\%. ${ }^{1} \mathrm{H}$ NMR (300 MHz, $\mathrm{CDCl}_{3}, J$ in $\left.\mathrm{Hz}\right) \delta 8.17\left(\mathrm{~d}, 4 \mathrm{H},{ }^{3} J=8.3, \mathrm{Ar} \mathrm{H}\right), 7.82(\mathrm{~s}, 1 \mathrm{H}$, triazole $\mathrm{H}), 7.79(\mathrm{~s}, 1 \mathrm{H}$, triazole $\mathrm{H}), 7.42-7.38(\mathrm{~m}, 4 \mathrm{H}, \mathrm{Ar} \mathrm{H})$, $5.62\left(\mathrm{~s}, 4 \mathrm{H}, 2 \mathrm{OCH}_{2}\right), 4.99-4.73\left(\mathrm{~m}, 5 \mathrm{H}, 2 \mathrm{NCH}_{2}, 1-\mathrm{H}\right), 3.85-3.72$ (m, 2H, 3-H, 6-H), 3.69-3.50 (m, 3H, 2-H, 4-H, 5-H), 3.35 (s, $\left.3 \mathrm{H}, \mathrm{OCH}_{3}\right), 2.60(\mathrm{bs}, 1 \mathrm{H}, \mathrm{OH}) ;{ }^{13} \mathrm{C} \mathrm{NMR}\left(75 \mathrm{MHz}, \mathrm{CDCl}_{3}\right) \delta$ $147.92,146.05,145.36,141.60,141.50,128.69,124.16,123.56$, $123.16,97.56,81.78,79.38,71.11,70.22,70.08,65.75,63.99$, 61.79, 55.01, 52.99; IR (KBr) v 3406, 3142, 3078, 2925, 1608, $1527,1522,1461,1348,1225,1189,1110,1050 \mathrm{~cm}^{-1}$; MS $(\mathrm{m} / \mathrm{z}): 649[\mathrm{M}+\mathrm{Na}]^{+}, 595\left[\mathrm{M}-\mathrm{CH}_{3} \mathrm{O}\right]^{+}$.

$(3 S, 4 R, 5 R, 6 R)-4,5-B i s((1-(4-f l u o r o b e n z y l)-1 H-1,2,3-t r i-$ azol-4-yl)methoxy)-2-(hydroxymethyl)-6-methoxy-tetrahydro2H-pyran-3-ol (5h): Syrup. Yield: 99\%. ${ }^{1} \mathrm{H}$ NMR (300 MHz, $\mathrm{CDCl}_{3}, J$ in $\left.\mathrm{Hz}\right) \delta 7.65(\mathrm{~s}, 1 \mathrm{H}$, triazole $\mathrm{H}), 7.53(\mathrm{~s}, 1 \mathrm{H}$, triazole $\mathrm{H}), 7.32-7.22$ (m, 4H, Ar H), 7.15-7.02 (m, 4H, Ar H), 5.55 (s, $\left.2 \mathrm{H}, \mathrm{OCH}_{2}\right), 5.46\left(\mathrm{~s}, 2 \mathrm{H}, \mathrm{OCH}_{2}\right), 4.99-4.83\left(\mathrm{~m}, 4 \mathrm{H}, 2 \mathrm{NCH}_{2}\right)$, $4.75\left(\mathrm{~d}, 1 \mathrm{H},{ }^{3} \mathrm{~J}=3.5,1-\mathrm{H}\right), 3.83-3.75$ (m, 3H, 5-H, 6-H), 3.623.59 (m, 2H, 3-H, 4-H), 3.54-3.50 (m, 1H, 2-H), 3.33 (s, 3H, $\left.\mathrm{OCH}_{3}\right), 2.99(\mathrm{bs}, 2 \mathrm{H}, 2 \mathrm{OH}) ;{ }^{13} \mathrm{C} \mathrm{NMR}\left(75 \mathrm{MHz}, \mathrm{CDCl}_{3}\right) \delta$ 164.11, 161.77, 160.81, 158.48, 145.59, 144.74, 130.61, 130.51, $130.25,129.73,129.62,124.42,122.64,122.43,121.66,121.48$, $121.29,115.84,115.55,115.27,97.38,81.83,79.31,79.15$, 70.56, 70.44, 65.27, 64.02, 62.16, 54.57, 53.07, 52.96, 47.30; $\operatorname{MS}(\mathrm{m} / \mathrm{z}): 595[\mathrm{M}+\mathrm{Na}]^{+}$.

(3S,4R,5R,6R)-4,5-Bis((1-(4-bromobenzyl)-1H-1,2,3-triazol-4-yl)methoxy)-2-(hydroxymethyl)-6-methoxy-tetrahydro2H-pyran-3-ol (5i): White solid. mp 66 - $68{ }^{\circ} \mathrm{C}$. Yield: $98 \%$. ${ }^{1} \mathrm{H}$ NMR (300 MHz, $\mathrm{CDCl}_{3}, J$ in $\left.\mathrm{Hz}\right) \delta 7.63(\mathrm{~s}, 1 \mathrm{H}$, triazole $\mathrm{H})$, $7.53(\mathrm{~s}, 1 \mathrm{H}$, triazole $\mathrm{H}), 7.48\left(\mathrm{t}, 4 \mathrm{H},{ }^{3} J=5.9\right.$, Ar H), $7.14(\mathrm{~d}$, $\left.2 \mathrm{H},{ }^{3} \mathrm{~J}=7.9, \mathrm{Ar} \mathrm{H}\right), 5.45\left(\mathrm{~s}, 4 \mathrm{H}, 2 \mathrm{OCH}_{2}\right), 4.96\left(\mathrm{~d}, 1 \mathrm{H},{ }^{3} \mathrm{~J}=\right.$ $\left.12.8,1 / 2 \mathrm{NCH}_{2}\right), 4.83-4.78\left(\mathrm{~m}, 3 \mathrm{H}, 3 / 2 \mathrm{NCH}_{2}, 1-\mathrm{H}\right), 3.83(\mathrm{~s}, 2 \mathrm{H}$, 6-H), 3.75 (d, $\left.2 \mathrm{H},{ }^{3} \mathrm{~J}=4.8,5-\mathrm{H}\right), 3.60\left(\mathrm{~d}, 2 \mathrm{H},{ }^{3} \mathrm{~J}=4.4,2-\mathrm{H}\right.$, 3-H), 3.52-3.49 (m, 1H, 4-H), 3.35 (s, 3H, OCH $), 2.99$ (bs, 2H, $2 \mathrm{OH}) ;{ }^{13} \mathrm{CNMR}\left(100 \mathrm{MHz}, \mathrm{CDCl}_{3}\right) \delta 146.09,145.41,133.70$, 133.48, 132.42, 129.94, 123.16, 122.46, 97.83, 82.30, 79.60, $71.16,70.87,65.86,64.45,62.58,55.19,53.73,53.60 ; \mathrm{MS}$ $(\mathrm{m} / z): 595[\mathrm{M}+\mathrm{Na}]^{+}, 573[\mathrm{M}+\mathrm{H}]^{+}$

$(3 R, 4 S, 5 S, 6 R)-2-(H y d r o x y m e t h y l)-6-m e t h o x y-4,5-b i s((1-$ octyl-1 $H$-1,2,3-triazol-4-yl)methoxy)-tetrahydro- $2 H$-pyran3-ol (8a): White solid. mp 44 - $45{ }^{\circ} \mathrm{C}$. Yield: $95 \%$. ${ }^{1} \mathrm{H}$ NMR $\left(300 \mathrm{MHz}, \mathrm{CDCl}_{3}, J\right.$ in $\left.\mathrm{Hz}\right) \delta 7.67(\mathrm{~s}, 1 \mathrm{H}$, triazole $\mathrm{H}), 7.64(\mathrm{~s}$, $1 \mathrm{H}$, triazole $\mathrm{H}), 5.06-4.80\left(\mathrm{~m}, 5 \mathrm{H}, 2 \mathrm{OCH}_{2}, 1-\mathrm{H}\right), 4.35-4.30(\mathrm{~m}$, $\left.4 \mathrm{H}, 2 \mathrm{NCH}_{2}\right), 3.85-3.81$ (m, 3H, 3-H, 6-H), 3.65-3.54 (m, 3H, 2-H, 4-H, 5-H), 3.39 (s, 3H, OCH $), 2.93$ (bs, 2H, 2OH), 1.88 (d, $\left.4 \mathrm{H},{ }^{3} \mathrm{~J}=5.4,2 \mathrm{CH}_{2}\right), 1.28\left(\mathrm{~s}, 20 \mathrm{H}, 10 \mathrm{CH}_{2}\right), 0.87\left(\mathrm{t}, 6 \mathrm{H},{ }^{3} \mathrm{~J}=6.8\right.$, $\left.2 \mathrm{CH}_{3}\right) ;{ }^{13} \mathrm{C} \mathrm{NMR}\left(75 \mathrm{MHz}, \mathrm{CDCl}_{3}\right) \delta 145.26,144.58,122.79$, 122.27, 97.80, 82.10, 79.52, 71.08, 70.63, 65.72, 64.45, 62.34, 54.99, 50.44, 50.34, 31.63, 30.22, 30.16, 28.97, 28.89, 26.44, 22.52, 13.98; IR (KBr) v 3139, 2924, 2856, 2598, 2363, 1600, 1577, 1458, 1435, 1408, 1321, 1300, 1224, 1169, 1069, 1004 $\mathrm{cm}^{-1}$; MS $(\mathrm{m} / \mathrm{z}): 603[\mathrm{M}+\mathrm{Na}]^{+}, 581[\mathrm{M}+\mathrm{H}]^{+}$.

4,5-Bis((1-dodecyl-1H-1,2,3-triazol-4-yl)methoxy)-2-(hydroxymethyl)-6-methoxy-tetrahydro-2H-pyran-3-ol (8b): White solid. mp $81-82{ }^{\circ} \mathrm{C}$. Yield: $98 \%$. ${ }^{1} \mathrm{H}$ NMR (300 MHz, $\mathrm{CDCl}_{3}$, $J$ in $\mathrm{Hz}) \delta 7.67(\mathrm{~s}, 1 \mathrm{H}$, triazole $\mathrm{H}), 7.64(\mathrm{~s}, 1 \mathrm{H}$, triazole $\mathrm{H})$, 5.02-4.81 (m, 5H, 2OCH, $1-\mathrm{H}), 4.32\left(\mathrm{t}, 4 \mathrm{H},{ }^{3} \mathrm{~J}=5.9,2 \mathrm{NCH}_{2}\right)$, 3.85-3.79 (m, 3H, 5-H, 6-H), 3.69-3.64 (m, 2H, 3-H, 4-H,), $3.54\left(\mathrm{dd}, 1 \mathrm{H},{ }^{3} \mathrm{~J}=3.6,{ }^{3} \mathrm{~J}=9.2,2-\mathrm{H}\right), 3.39\left(\mathrm{~s}, 3 \mathrm{H}, \mathrm{OCH}_{3}\right), 1.89$ (s, $\left.4 \mathrm{H}, 2 \mathrm{CH}_{2}\right), 1.30-1.25\left(\mathrm{~m}, 36 \mathrm{H}, 18 \mathrm{CH}_{2}\right), 0.87\left(\mathrm{t}, 6 \mathrm{H},{ }^{3} \mathrm{~J}=5.7\right.$, $\left.2 \mathrm{CH}_{3}\right) ;{ }^{13} \mathrm{C} \mathrm{NMR}\left(75 \mathrm{MHz}, \mathrm{CDCl}_{3}\right) \delta 145.29,144.57,122.82$, 122.34, 97.79, 82.10, 79.47, 71.12, 70.54, 65.78, 64.47, 62.20, 54.97, 50.46, 50.33, 31.82, 30.24, 30.17, 29.53, 29.46, 29.33, 29.26, 28.95, 26.45, 22.60, 14.05; IR (KBr) v 3139, 2924, 2856, 2598, 2363, 1600, 1577, 1458, 1435, 1408, 1321, 1300, 1224, 1169, 1069, $1004 \mathrm{~cm}^{-1}$; MS $(\mathrm{m} / \mathrm{z}): 715[\mathrm{M}+\mathrm{Na}]^{+}$

Antibacterial and antifungal assays. The in vitro minimal inhibitory concentrations (MICs) of the target compounds were 
determined by broth microdilution assay method in 96-well microtest plates. ${ }^{30}$ The bacterial suspension was adjusted with sterile saline to a concentration of $1 \times 10^{5} \mathrm{CFU}$. The tested compounds were dissolved in DMSO to prepare the stock solutions. The tested compounds and reference drugs were prepared in Mueller-Hinton broth (Guangdong huaikai microbial sci. \& tech co., Ltd, Guangzhou, Guangdong, China) by twofold serial dilution to obtain the required concentrations of 512, 256, 128, $64,32,16,8,4,2,1,0.5 \mu \mathrm{g} / \mathrm{mL}$. These dilutions were inoculated and incubated at $37^{\circ} \mathrm{C}$ for $24 \mathrm{~h}$. To ensure that the solvent had no effect on bacterial growth, a control test was performed with test medium supplemented with DMSO at the same dilutions as used in the experiment. The tested microorganism strains were provided by the School of Pharmaceutical Sciences, Southwest University. Fluconazole and Chloramphenicol were obtained from their respective manufacturers served as controls.

All compounds were evaluated for their antibacterial activity against $S$. aureus (ATCC29213) and B. subtilis as Gram-positive, E. coli (ATCC25922), P. aeruginosa and B. proteus as Gram-negative bacteria, as well as their antifungal activity against $C$. albicans (ATCC 76615) and $A$. fumigatus, at the concentrations of the antimicrobial agents ranging from $0.5 \mu \mathrm{g} / \mathrm{mL}$ to $512 \mu \mathrm{g} / \mathrm{mL}$ and scored for $\mathrm{MIC}_{50}$ as the level of growth inhibition of the tested microorganisms. The minimum inhibitory concentration (MIC) values (in $\mathrm{mM}$ ) were summarized in Table 2.

Acknowledgments. This work was partially supported by Natural Science Foundation of Chongqing (CSCT: 2007BB5369, 2009BB5296) and Southwest University (SWUB2006018, XSGX0602).

\section{References}

1. DeMarco, C. E.; Cushing, L. A.; Frempont-Manso, E. Antimicrob. Agents Chemother. 2007, 51, 3235.

2. Moses, J. E.; Moorhouse, A. D. Chem. Soc. Rev. 2007, 36, 1249.

3. Hawker, C. J.; Wooley, K. L. Science 2005, 309, 1200.

4. Lewis, W. G.; Green, L. G.; Grynszpan, F.; Radić, Z.; Carlier, P. R.; Taylor, P.; Finn, M. G.; Sharpless, K. B. Angew Chem. Int. Ed. 2002, 41, 1053.

5. Kolb, H. C.; Finn, M. G.; Sharpless, K. B. Angew Chem. Int. Ed. 2001, 40, 2004.

6. Wu, J. J.; Green, N.; Hotchandani, R.; Hu, Y. H.; Condon, J.; Huang, A.; Kaila, N.; Li, H. Q.; Guler, S.; Li, W.; Tam, S. Y.; Wang, Q.; Pelker, J.; Marusic, S.; Hsu, S.; Hall, J. P.; Telliez, J. B.; Cui, J. Q.; Lin, L. L. Bioorg. Med. Chem. Lett. 2009, 19, 3485.
7. Boddy, I. K.; Briggs, G. G.; Harrison, R. P.; Jones, T. H.; ÓMahony, M. J.; Marlow, I. D.; Roberts, B. G.; Willis, R. J.; Bardsley, R.; Reid, J. Pestic. Sci. 1996, 48, 189.

8. Phillips, O. A.; Udo, E. E.; Abdel-Hamid, M. E.; Varghese, R. Eur. J. Med. Chem. 2009, 44, 3217.

9. Aher, N. G.; Pore, V. S.; Mishra, N. N.; Kumar, A.; Shukla, P. K.; Sharma, A.; Bhat, M. K. Bioorg. Med. Chem. Lett. 2009, 19, 759.

10. Gill, C.; Jadhav, G.; Shaikh, M.; Kale, R.; Ghawalkar, A.; Nagargoje, D.; Shiradkar, M. Bioorg. Med. Chem. Lett. 2008, 18, 6244.

11. Giffin, M. J.; Heaslet, H.; Brik, A; Lin, Y. C.; Cauvi, G.; Wong, C. H.; McRee, D. E.; Elder, J. H.; Stout, C. D.; Torbett, B. E. J. Med. Chem. 2008, 51, 6263 .

12. Whiting, M.; Muldoon, J.; Lin, Y. C.; Silverman, S. M.; Lindstrom, W.; Olson, A. J.; Kolb, H. C.; Finn, M. G.; Sharpless, K. B.; Elder, J. H.; Fokin, V. V. Angew Chem. Int. Ed. 2006, 45, 1435.

13. Tron, G. C.; Pirali, T.; Billington, R. A.; Canoniico, P. L.; Sorba, G.; Genazzani, A. A. Med. Res. Rev. 2008, 28, 278.

14. Somu, R. V.; Boshoff, H.; Qiao, C. H.; Bennett, E. M.; Barry, C. E.; Aldrich, C. C. J. Med. Chem. 2006, 49, 31.

15. Danoun, S.; Baziard-Mouysset, G.; Stigliani, J.; Payard, M.; Selkti, M.; Tomas, V. A. Heterocycl. Commun. 1998, 4, 45.

16. Biagi, G.; Calderone, V.; Giorgi, I.; Livi, O.; Martinotti, E.; Martelli, A.; Nardi, A. Il Farmaco 2004, 59, 397.

17. Hou, D. R.; Alam, S.; Kuan, T. C.; Ramanathan, M.; Lin, T. P.; Hung, M. S. Bioorg. Med. Chem. Lett. 2009, 19, 1022.

18. Collin, M.-P.; Hobbie, S. N.; Böttger, E. C.; Vasella, A. Helv. Chim. Acta. 2008, 91, 1838 .

19. Horne, W. S.; Yadav, M. K.; Stout, C. D.; Ghadiri, M. R. J. Am. Chem. Soc. 2004, 126, 15366.

20. Huber, D.; Hubner, H.; Gmeiner, P. J. Med. Chem. 2009, 52, 6860.

21. Pokrovskaya, V.; Belakhov, V.; Hainrichson, M.; Yaron, S.; Baasov, T. J. Med. Chem. 2009, 52, 2243.

22. Akri, K. E.; Bougrin, K.; Balzarini, J.; Faraj, A.; Benhida, R. Bioorg. Med. Chem. Lett. 2007, 17, 6656.

23. Karthikeyan, M. S.; Holla, B. S.; Kumari, N. S. Eur. J. Med. Chem. 2008, 43, 309.

24. Chai, X. Y.; Zhang, J.; Song, Y. L.; Hu, H. G.; Zou, Y.; Zhao, Q. J.; Dan, Z. G.; Zhang, D. Z.; Wu, Q. Y. Bioorg. Med. Chem. Lett. 2009, 19. 1811.

25. Sheng, C. Q.; Zhang, W. N.; Ji, H. T.; Zhang, M.; Song, Y. L.; Xu, H.; Zhu, J.; Miao, Z. Y.; Jiang, Q. F.; Yao, J. Z.; Zhou, Y. J.; Zhu, J.; Lu, J. G. J. Med. Chem. 2006, 49, 2512.

26. Rezaei, Z.; Khabnadideh, S.; Pakshir, K.; Hossaini, Z.; Amiri, F.; Assadpour, E. Eur. J. Med. Chem. 2009, 44, 3064.

27. Amblard, F.; Cho, J. H.; Schinazi, R. F. Chem. Rev. 2009, 109, 4207.

28. Thibodeaux, C. J.; Melancon, C. E.; Liu, H. Nature 2007, 446, 1008.

29. Dwek, R. A.; Butters, T. D. Chem. Rev. 2002, 102, 283.

30. Kadi, A. A.; El-Brollosy, N. R.; Al-Deeb, O. A.; Habib, E. E.; Ibrahim, T. M.; El-Emam, A. A. Eur. J. Med. Chem. 2007, 42, 235.

31. Tankam, P. F.; Mischnick, P.; Hopf, H.; Jones. P. G. Carbohyd. Res. 2007, 342, 2031. 